Prepared For SUBMission to JCAP

\title{
Neutrino constraints: what large-scale structure and CMB data are telling us?
}

Matteo Costanzi, ${ }^{a, b}$ Barbara Sartoris ${ }^{a, b}$ Matteo Viel, ${ }^{c, b}$ Stefano Borgani $^{a, b, c}$

\footnotetext{
${ }^{a}$ Astronomy Unit, Department of Physics, University of Trieste, via Tiepolo 11, I-34143 Trieste, Italy

${ }^{b}$ INFN-National Institute for Nuclear Physics, via Valerio 2, I-34127 Trieste, Italy

${ }^{c}$ INAF-Osservatorio Astronomico di Trieste, via Tiepolo 11, I-34143 Trieste, Italy
} 
E-mail: costanzi@oats.inaf.it, sartoris@oats.inaf.it,viel@oats.inaf.it, borgani@oats.inaf.it

Abstract. We discuss the reliability of neutrino mass constraints, either active or sterile, from the combination of different low redshift Universe probes with measurements of CMB anisotropies. In our analyses we consider WMAP 9-year or Planck Cosmic Microwave Background (CMB) data in combination with Baryonic Acoustic Oscillations (BAO) measurements from BOSS DR11, galaxy shear measurements from CFHTLenS, SDSS Ly- $\alpha$ forest constraints and galaxy cluster mass function from Chandra observations. At odds with recent similar studies, to avoid model dependence of the constraints we perform a full likelihood analysis for all the datasets employed. As for the cluster data analysis we rely on to the most recent calibration of massive neutrino effects in the halo mass function and we explore the impact of the uncertainty in the mass bias and re-calibration of the halo mass function due to baryonic feedback processes on cosmological parameters. We find that none of the low redshift probes alone provide evidence for massive neutrino in combination with CMB measurements, while a larger than $2 \sigma$ detection of non zero neutrino mass, either active or sterile, is achieved combining cluster or shear data with CMB and BAO measurements. Yet, the significance of the detection exceeds $3 \sigma$ if we combine all four datasets. For a three active neutrino scenario, from the joint analysis of $\mathrm{CMB}, \mathrm{BAO}$, shear and cluster data including the uncertainty in the mass bias we obtain $\sum m_{\nu}=0.29_{-0.21}^{+0.18} \mathrm{eV}$ and $\sum m_{\nu}=0.22_{-0.18}^{+0.17} \mathrm{eV}$ (95\%CL) using WMAP9 or Planck as CMB dataset, respectively. The preference for massive neutrino is even larger in the sterile neutrino scenario, for which we get $m_{s}^{\text {eff }}=0.44_{-0.26}^{+0.28} \mathrm{eV}$ and $\Delta N_{\text {eff }}=0.78_{-0.59}^{+0.60}(95 \% \mathrm{CL})$ from the joint analysis of Planck, BAO, shear and cluster datasets. For this data combination the vanilla $\Lambda$ CDM model is rejected at more than $3 \sigma$ and a sterile neutrino mass as motivated by accelerator anomaly is within the $2 \sigma$ errors. Conversely, the Ly- $\alpha$ data favour vanishing neutrino masses and from the data combination Planck+BAO + Ly- $\alpha$ we get the tight upper limits $\sum m_{\nu}<0.14 \mathrm{eV}$ and $m_{s}^{\text {eff }}<0.22 \mathrm{eV}-$ $\Delta N_{\text {eff }}<1.11$ (95\%CL) for the active and sterile neutrino model, respectively. Finally, results from the full data combination reflect the tension between the $\sigma_{8}$ constraints obtained from cluster and shear data and that inferred from Ly- $\alpha$ forest measurements; in the active neutrino scenario for both CMB datasets employed, the full data combination yields only an upper limits on $\sum m_{\nu}$, while assuming an extra sterile neutrino we still get preference for non-vanishing mass, $m_{s}^{\text {eff }}=0.26_{-0.24}^{+0.22} \mathrm{eV}$, and dark contribution to the radiation content, $\Delta N_{\text {eff }}=0.82 \pm 0.55$.

Keywords: cosmology: large-scale structure of Universe; neutrinos; galaxies: clusters. 


\section{Contents}

1 Introduction 1

2 Cosmological data analysis $\quad 2$

2.1 Models 2

2.2 Data and analysis 3

3 Results $\quad 5$

$\begin{array}{lll}3.1 & \text { Massive neutrinos } & 7\end{array}$

$\begin{array}{lll}3.2 & \text { Extra sterile massive neutrinos } & 10\end{array}$

4 Conclusions

\section{Introduction}

Results from Planck measurements of the cosmic microwave background (CMB) temperature anisotropies have yielded sub-percent level constraints on the cosmological parameters of the concordance $\Lambda$ CDM model [1]. The Planck CMB data by themselves appear to be well described by the six standard $\Lambda$ CDM parameters, and show no preference for extended models. However, in the framework of the $\Lambda \mathrm{CDM}$ model, several probes of the low redshift Universe exhibit tension with the Planck results. In particular, Planck finds a larger and more precise value of the matter density at recombination than previous CMB data; this results in a lower value for the current expansion rate $H_{0}$, and a higher value of the matter density fluctuations $\sigma_{8}$. These changes lead to a $\sim 2-3 \sigma$ tension with direct measurements of $H_{0}[2]$ and $\sigma_{8}$ measurements from galaxy shear power spectrum [3], galaxy-galaxy lensing [4], redshift space distortion (RSD) [5] and clusters abundance [6-9]. Meanwhile, agreement with distance measurements from Baryon Acoustic Oscillations (BAO) suggests that the discrepancy cannot be resolved involving exotic dark energy models or curvature which modify the recent expansion history.

Beside unresolved systematic effects neutrinos can offer a possible means to relieve this tension. Sterile neutrinos change the expansion rate at recombination and hence the calibration of the standard ruler with which CMB and BAO observations infer distances, while massive neutrino, either sterile or active, suppress small scale clustering at late time, relieving the tension with $\sigma_{8}$ measurements [see 10-12, for reviews]. Massive neutrinos are an appealing solution since oscillation experiments with solar and atmospheric neutrinos have already provided evidence for their mass, with room for extra sterile species, supported by anomalies in short baseline and reactor neutrino experiments [13-16]. A number of studies have carried out joint analyses of various data combinations finding that a neutrino mass of $0.3-0.4 \mathrm{eV}$ provides a better fit to CMB data with low redshift Universe measurements than the vanilla $\Lambda$ CDM model [17-24], although these conclusions are not universally accepted [25-27]. While, none of these low redshift datasets combined individually with CMB measurements provide strong evidence for non-zero neutrino masses, the hint for neutrino mass is driven mainly by low redshift growth of structure constraints (e.g. from shear and RSD measurements or cluster number counts). 
In particular, galaxy clusters offer a powerful complementary probe to the CMB and geometric probes as BAO thanks to the tight constraints provided on the so called cluster normalization condition, $\sigma_{8} \Omega_{\mathrm{m}}^{\gamma}$ [see 8, 28, for reviews in cluster cosmology]. X-ray [6, 29], Sunyaev-Zel'Dovich [9, 30, 31] and optical [7] cluster surveys yield consistent results favouring a value for the cluster normalization condition lower than the value derived from Planck data. This tension between Planck and cluster data and the combination with BAO results could be taken as an evidence for non-vanishing neutrino masses. However, the robustness of such constraints from cluster number counts depend on our capability to recover cluster masses from proxies and to have precise theoretical predictions for the spatial number density of halos (the halo mass function, HMF) [32-34]. Thus it is worth to investigate possible sources of systematic errors in cluster data which could lead to misinterpretation of the results and to assess which combination of low-redshift datasets with CMB data prefers a non-zero neutrino mass within a given cosmological model.

In this work we derive constraints on neutrino properties combining WMAP9 [35] or Planck [1] CMB data with several probes of the local Universe: BOSS DR11 BAO scale [36] and CFTHLenS shear [3] measurements, SDSS Ly- $\alpha$ forest power spectrum constraints [37] and cluster mass function from Chandra observations [38]. We consider two possible extensions of the standard $\Lambda \mathrm{CDM}$ model: a scenario with three degenerate active neutrinos, or a sterile massive neutrino model with three active neutrinos distributed as in the minimal normal hierarchy scenario. So far, many works [e.g. 19, 20, 22, 23] derived neutrino mass constraints including cluster and/or shear constraints obtained within a $\Lambda$ CDM model. In order to avoid misleading results due to model dependence of the constraints we perform a full likelihood analysis for all the datasets employed in this work, without doing a sampling of the posterior probability (as in e.g. [18]). In particular for the clusters data analyses we take into account the effect of possible bias in the mass estimation and adopt different prescriptions for the HMF. As for the latter, we consider the correction to the HMF proposed by [39] and [40] for cosmology with massive neutrinos. Moreover, we investigate how the different calibration of the HMF due to baryonic feedback processes presented in [41] affects the cosmological constraints.

The paper is organized as follows. Section 2 describes the cosmological models and datasets used in this work. In section 3 we present and discuss our results . Finally, we draw the main conclusions in section 4 .

\section{Cosmological data analysis}

\section{$2.1 \quad$ Models}

The baseline scenario analysed in this work is a $\Lambda$ CDM model with three degenerate massive neutrinos, defined by the parameters:

$$
\left\{\Omega_{\mathrm{c}} h^{2}, \Omega_{\mathrm{b}} h^{2}, \Theta_{\mathrm{s}}, \tau, n_{\mathrm{s}}, \log \left(10^{10} A_{\mathrm{s}}\right), \sum m_{\nu}\right\},
$$

with $\Omega_{\mathrm{c}} h^{2}$ and $\Omega_{\mathrm{b}} h^{2}$ being the physical cold dark matter and baryon energy densities, $\Theta_{\mathrm{s}}$ the ratio between the sound horizon and the angular diameter distance at decoupling, $\tau$ the Thomson optical depth at reionization, $n_{\mathrm{s}}$ the scalar spectral index, $A_{\mathrm{s}}$ the amplitude of the primordial power spectrum and $\sum m_{\nu}$ the total neutrino mass. Note that given the current precision of cosmological constraints from available data, the effect of mass splitting is negligible and the degenerate model can be assumed without loss of generality [see e.g. 
42]. We then consider a scenario with a massive sterile neutrino component which has been suggested as a possible solution for the reactor [43], Gallium [44, 45] and accelerator [46] anomalies in neutrino oscillation experiments. Reactor and Gallium experiments prefer a new mass squared difference of $\Delta m^{2} \gtrsim 1 \mathrm{eV}^{2}$, while various accelerator experiments constrain $\Delta m^{2}$ to be $\sim 0.5 \mathrm{eV}^{2}$ (see [16] and reference therein). For this model we assume one massive active neutrino with $\sum m_{\nu}=0.06 \mathrm{eV}$ (the minimum mass allowed by neutrino oscillation experiments) and we introduce two parameters to describe the extra hot relic component: the effective number of extra relativistic degree of freedom $\Delta N_{\text {eff }}$ and the effective sterile neutrino mass $m_{\mathrm{s}}^{\text {eff }}$. The former parametrizes any contribution to the radiation energy content $\left(\rho_{\mathrm{r}}\right)$ besides photons in the radiation dominated era through the formula:

$$
\rho_{\mathrm{r}}=\left[1+\frac{7}{8}\left(\frac{4}{11}\right)^{4 / 3} N_{\mathrm{eff}}\right] \rho_{\gamma} .
$$

In the standard model $N_{\text {eff }}=3.046$ accounts for the three active neutrino species; thus $\Delta N_{\text {eff }}=N_{\text {eff }}-3.046>0$ indicates new physics beyond the standard model: an extra thermalised light fermion would contribute $\Delta N_{\text {eff }}=1$, but more generally a non-integer $\Delta N_{\text {eff }}$ value could arise from different physical phenomena, such as lepton asymmetries [47], partial thermalisation of new fermions [48], particle decay [49], non-thermal production of dark matter [50,51], gravity waves [52] or early dark energy [53]. The large values of the mass squared difference and mixing angles invoked to resolve reactor, Gallium $\left(\sin ^{2} 2 \Theta \gtrsim 0.1\right)$ and accelerator $\left(\sin ^{2} 2 \Theta \sim 5 \times 10^{-3}\right)$ anomalies suggest a fully thermalisation of the sterile neutrino in the early Universe [54], and thus a contribution of $\Delta N_{\text {eff }}=1$ to the dark radiation. The parameter $m_{\mathrm{s}}^{\mathrm{eff}}$, in the case of thermally-distributed sterile neutrino, is related to the physical mass $m_{\mathrm{s}}$ via

$$
m_{\mathrm{s}}^{\mathrm{eff}}=\left(T_{\mathrm{s}} / T_{\nu}\right)^{3} m_{\mathrm{s}}=\left(\Delta N_{\mathrm{eff}}\right)^{3 / 4} m_{\mathrm{s}}
$$

where $T_{\mathrm{S}}$ and $T_{\nu}$ represent the current temperature of the sterile and active neutrinos, respectively. Alternatively, if the sterile neutrino is distributed proportionally to the active species due to oscillations the physical mass can be expressed as $m_{\mathrm{s}}^{\text {eff }}=\left(\Delta N_{\text {eff }}\right) m_{\mathrm{s}}$, which corresponds to the Dodelson-Widrow scenario [55]. In both cases for a fully thermalised sterile neutrino, $\Delta N_{\text {eff }}=1$, one gets $m_{\mathrm{s}}^{\text {eff }}=m_{\mathrm{s}}$. In our analysis we adopt the prior $m_{\mathrm{s}}^{\text {eff }} /\left(\Delta N_{\text {eff }}\right)^{3 / 4}<10 \mathrm{eV}$ to avoid a degeneracy between very massive neutrinos and cold dark matter.

\subsection{Data and analysis}

We infer posterior probability distributions by means of the Monte Carlo Markov Chain technique using the publicly available code CosmoMC ${ }^{1}$ [56] for various combinations of the following datasets:

$C M B$ - We consider CMB temperature and polarization measurements from 9-year WMAP data release (hereafter WMAP9) [35] or, alternatively, temperature power spectrum from the Planck satellite [1] combined with large-scale TE- and EE-polarization power spectra from WMAP9 (hereafter Planck). These datasets are analysed using the likelihood functions provided by the Planck collaboration [57], and publicly available at Planck Legacy Archive ${ }^{2}$ and marginalizing over the foreground nuisance parameters. The helium abundance is computed as a function of $\Omega_{\mathrm{b}} h^{2}$ and $N_{\text {eff }}$, following the Big Bang Nucleosynthesis theoretical

\footnotetext{
${ }^{1}$ http://cosmologist.info/cosmomc/

${ }^{2}$ http://pla.esac.esa.int/pla/aio/planckProducts.html
} 
predictions. In the Planck analysis we fix the lensing spectrum normalization parameter to $A_{\mathrm{L}}=1$, if not otherwise stated. The WMAP9 dataset is not sensitive to the gravitational lensing signal since its effects can be detected only at large multipoles.

$B A O$ - We include the most recent and accurate measurements of the BAO scales from BOSS Data Release 11 [36]. Exploiting a sample of nearly one million galaxies observed over 8500 square degree between redshift $0.2<z<0.7$, DR11 results provides percent level constraints on the peak position of the spherically averaged galaxy correlation function at redshift $z=0.32$ and $z=0.57$. The likelihood function associated to this dataset is estimated using the likelihood code distributed with the CosmoMC package.

Shear - We use the 6-bin tomography angular galaxy shear power spectra data from the CFTHLenS survey $[3]^{3}$. The survey spans over 154 square degrees in five optical bands, with shear and photometric redshift measurements for a galaxy sample with a median redshift of $z=0.70$. Constraints from this datasets are derived using a modified version of the CosmoMC module ${ }^{4}$ for the weak lensing COSMOMS 3D data [58]. The code has been substantially modified in order to reproduce the analysis described in [3] which makes use of 21 sets of cosmic shear correlation functions associated to 6 redshift bins, each spanning the angular range of $1.5-35$ arcmin, to extract cosmological information. As in [3], we also include in the module the model for the intrinsic alignment treatment developed by [59], which accounts for both intrinsic alignment of physically nearby galaxies and the shear-shape correlation for galaxies separated by large physical distances along the line of sight. This model, which is based on a fitting approach, has the advantage of needing only one additional nuisance parameter, marginalized over in the analysis, to predict both the intrinsic alignment contributions to the shear correlation functions. We verified that our module reproduces well the results presented in [3] for a $\Lambda \mathrm{CDM}$ model.

$L y-\alpha$ - We rely on the SDSS Ly- $\alpha$ forest data from [37] to constrain the amplitude, slope and curvature of the linear matter power spectrum at scale $k=0.009 \mathrm{~s} \mathrm{~km}^{-1}$ and redshift $z=3$. We combine this dataset by implementing the Ly- $\alpha$ likelihood code distributed with the CosmoMC package. The module has been updated to work with the new version of CosmoMC and it has been implemented with the patch written by A. Slosar ${ }^{5}$ in order to support extended model analysis. Note that this data set does not include the most recent BOSS data of [60] that will soon provide an updated value of the upper limits obtained in [61], by using a new technique to sample the parameter space [62] and hydro simulations that incorporate massive neutrinos [63].

Clusters - Constraints from galaxy clusters are obtained exploiting the CCCP catalogue presented in [38]. The catalogue consists of X-ray Chandra observations of 37 clusters with $\langle z\rangle=0.55$ derived from the $400 \mathrm{deg}^{2}$ ROSAT survey and 49 brightest $z \approx 0.05$ clusters detected in the ROSAT All-Sky Survey, which provide a robust determination of the cluster mass function at low and high redshifts. To derive cosmological constraints we developed our own module for CosmoMC following the fitting procedure outlined in [38]. For the cluster masses we use $Y_{x}$ proxy mass estimations [64], which allows us to implement the X-ray luminosity-mass relation presented in [38] needed to compute the survey volume as a function of the mass. The theoretical abundance of massive halo is computed using the Tinker HMF [65], where the coefficient of the fitting formula are obtained interpolating table 2 of [65] for halos with $\Delta_{\text {mean }}=\Delta_{\text {critical }} / \Omega_{\mathrm{m}}=500 / \Omega_{\mathrm{m}}$, according to the cluster mass

\footnotetext{
${ }^{3}$ http://cfhtlens.org/astronomers/cosmological-data-products

${ }^{4}$ http://www.astro.caltech.edu/ rjm/cosmos/cosmomc/

${ }^{5} \mathrm{http}: / /$ www.slosar.com/aslosar/lya.html
} 
definition of [38]. We verified that our analysis reproduces accurately the results of [66] for the combination of WMAP 7-year and CCCP cluster data. To properly take into account the effects of massive neutrinos on the HMF calibration, we neglect the weakly clustering neutrino component when calculating the halo mass, as suggested by many authors [e.g. 6769]. Moreover, following $[39,40]$ the variance of the matter perturbations, required to predict the HMF, is computed using only the cold dark matter and baryon linear power spectrum, in order to neglect the suppression of the matter density fluctuations on scales smaller than the neutrino free-streaming length. These corrections entail an increase of the HMF with respect to the previous calibration. This effect is larger for larger neutrino masses and higher number of massive neutrino species. In turn, the increase in the HMF affects the resulting constraints on cosmological parameters, e.g. by steepening the $\sigma_{8}-\Omega_{\mathrm{m}}$ degeneracy direction, thereby reducing the $\sigma_{8}$ mean value [70]. Besides these modifications to the original analysis of [38] we test the effect of other sources of systematics. To address the impact on clusters constraints due to baryonic feedback processes we implement the correction to the HMF proposed by [41]; the net effect of baryonic processes is to generate shallower density profiles and a corresponding decrease of halo masses with respect to the dark matter only case used to fit the HMF [e.g. 33, 65, 71-73]. This effect is taken into account correcting the halo masses through a mass dependent fit to the halo mass variation induced by baryonic processes. Similar studies have been carried out by different groups [74-77] that found consistent results (cf. also [77]).

The main source of systematic errors is related to the uncertainty in cluster mass measurements. For the catalogue used in this work cluster masses have been inferred by using a scaling relation between total mass and the product of hot intracluster gas mass and temperature, as inferred from X-ray observations. This scaling relation has been calibrated by resorting to $\mathrm{X}$-ray hydrostatic mass measurements for nearby galaxy clusters [see 38 , for details]. However, mass measurements from X-ray measurements suffer for different sources of possible systematic biases, e.g. associated to departures from spherical symmetry, to biases in X-ray measurement of gas temperature [e.g. 78, 79], or to violation of hydrostatic equilibrium due to the presence non-thermal pressure support [e.g. 80, 81].

To consider this uncertainty we introduce a nuisance bias parameter defined as $M^{e s t} / M^{\text {true }}=$ $B_{M}$, which is varied in the range $[0.8: 1.0]$ when included in the fit. This prior accounts for the constraints on the mass bias from [82] and [83] obtained comparing Chandra X-ray masses with MMT/Megacam weak lensing masses of 8 clusters between redshift $0.39-0.80$ and CLASH weak lensing masses of 20 clusters between redshift $0.2-0.5$, respectively. A different range for the mass bias, $B_{M} \simeq 0.7 \pm 0.1$, is suggested by the analysis by [84] which however uses X-ray masses derived from XMM-Newton temperature measurements. Temperature measurements from XMM-Newton could be systematically lower with respect to those obtained from Chandra observations, as discussed by [83, 85, 86], thus providing a larger mass bias.

\section{Results}

For each of the cases that we describe here below, we run four independent chains, requiring the fulfilment of the Gelman \& Rubin [87] criteria with $R-1 \leqslant 0.03$ as convergence test. The best fit values are obtained with the BOBYQA maximisation routine provided in CosmoMC. If not otherwise stated, errors and upper limits reported in the text have to be intended at 95\% confidence level. 

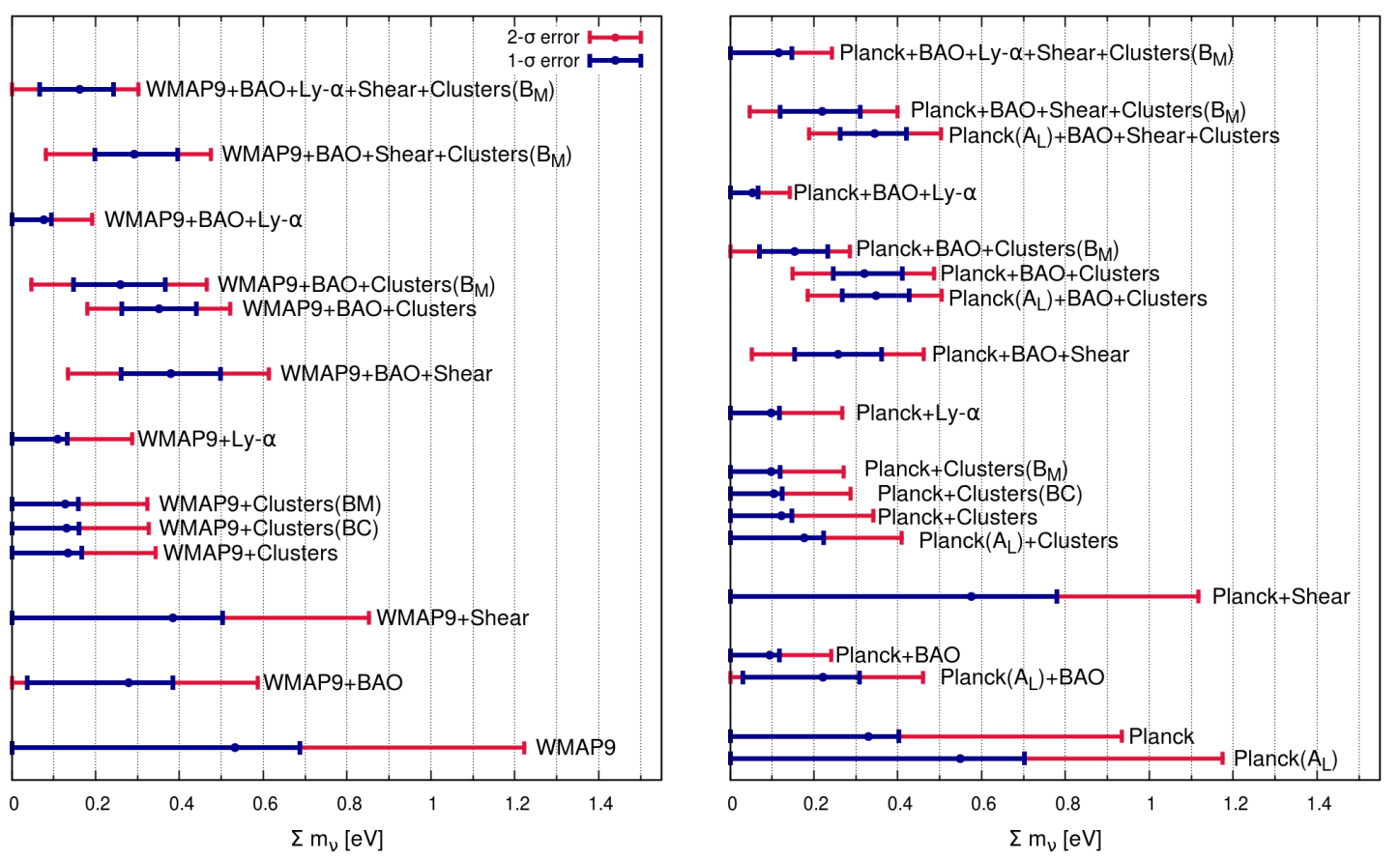

Figure 1: Summary of the $1 \sigma$ and $2 \sigma$ errors on $\sum m_{\nu}$ obtained from the dataset combinations discussed in section 3.1 within a $\Lambda \mathrm{CDM}+\sum m_{\nu}$ model.
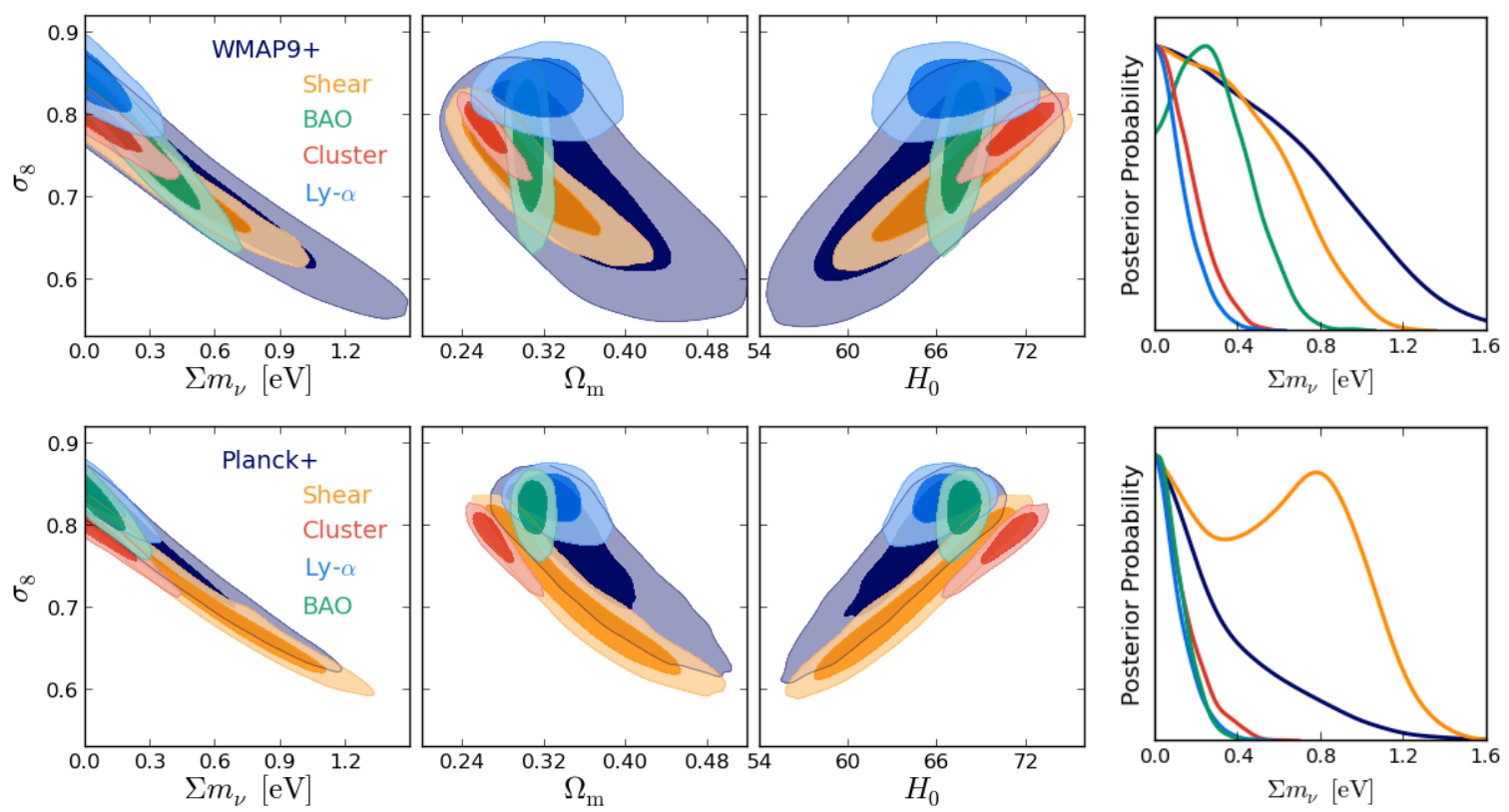

Figure 2: Left -Joint $68 \%$ and $95 \% \mathrm{CL}$ contours in the $\sigma_{8}-\left(\Omega_{\mathrm{m}}, H_{0}, \sum m_{\nu}\right)$ planes for a $\Lambda \mathrm{CDM}+\sum m_{\nu}$ model from the combination of WMAP9 (upper panels) or Planck (lower panels) data with different low redshift Universe probes. Right - Posterior probability distribution for $\sum m_{\nu}$ from the same data combination. 
Table 1: Constraints on $\Omega_{\mathrm{m}}, \sigma_{8}$ and $\sum m_{\nu}$ for a $\Lambda \mathrm{CDM}+\sum m_{\nu}$ model combining different datasets. Errors are reported at $68 \%$ confidence level for $\sigma_{8}$ and $\Omega_{\mathrm{m}}$, and both $68 \%$ and $95 \%$ confidence level for $\sum m_{\nu}$. Notations included in parenthesis denote modifications to the standard setting: $(\mathrm{BC})$ stands for the baryon correction to the HMF, while $\left(B_{M}\right)$ and $\left(A_{\mathrm{L}}\right)$ indicate analyses with the bias or lensing signal parameter marginalized out.

\begin{tabular}{|c|c|c|c|c|}
\hline \multirow[t]{2}{*}{ Dataset } & \multirow[t]{2}{*}{$\Omega_{\mathrm{m}}$} & \multirow[t]{2}{*}{$\sigma_{8}$} & \multicolumn{2}{|c|}{$\sum m_{\nu}[\mathrm{eV}]$} \\
\hline & & & $68 \% \overline{\mathrm{CL}}$ & $95 \% \mathrm{CL}$ \\
\hline WMAP9 & $0.347_{-0.079}^{+0.043}$ & $0.714_{-0.068}^{+0.091}$ & $<0.68$ & $<1.22$ \\
\hline WMAP9+Cluster & $0.264_{-0.017}^{+0.010}$ & $0.780_{-0.016}^{+0.028}$ & $<0.17$ & $<0.34$ \\
\hline WMAP9+Cluster $\left(B_{M}\right)$ & $0.275_{-0.020}^{+0.014}$ & $0.793_{-0.019}^{+0.029}$ & $<0.16$ & $<0.33$ \\
\hline WMAP9+Cluster(BC) & $0.271_{-0.017}^{+0.011}$ & $0.789_{-0.017}^{+0.026}$ & $<0.16$ & $<0.33$ \\
\hline WMAP9+BAO & $0.304_{-0.011}^{+0.009}$ & $0.759_{-0.043}^{+0.062}$ & $0.28_{-0.24}^{+0.11}$ & $<0.59$ \\
\hline WMAP9+Shear & $0.305_{-0.055}^{+0.029}$ & $0.726_{-0.046}^{+0.061}$ & $<0.50$ & $<0.85$ \\
\hline WMAP9+Ly- $\alpha$ & $0.320_{-0.033}^{+0.026}$ & $0.830_{-0.021}^{+0.025}$ & $<0.13$ & $<0.29$ \\
\hline WMAP9+BAO+Cluster & $0.298_{-0.009}^{+0.009}$ & $0.735_{-0.032}^{+0.015}$ & $0.35_{-0.09}^{+0.09}$ & $0.35_{-0.17}^{+0.17}$ \\
\hline WMAP9+BAO+Cluster $\left(B_{M}\right)$ & $0.298_{-0.009}^{+0.009}$ & $0.765_{-0.028}^{+0.024}$ & $0.26_{-0.11}^{+0.11}$ & $0.26_{-0.20}^{+0.20}$ \\
\hline WMAP9+BAO+Shear & $0.303_{-0.010}^{+0.010}$ & $0.724_{-0.028}^{+0.028}$ & $0.38_{-0.12}^{+0.12}$ & $0.38_{-0.24}^{+0.23}$ \\
\hline WMAP9+BAO $+\mathrm{Ly}-\alpha$ & $0.305_{-0.009}^{+0.009}$ & $0.833_{-0.019}^{+0.021}$ & $<0.09$ & $<0.19$ \\
\hline WMAP9+BAO+Cluster $\left(B_{M}\right)+$ Shear & $0.297_{-0.010}^{+0.010}$ & $0.752_{-0.022}^{+0.017}$ & $0.29_{-0.9}^{+0.11}$ & $0.29_{-0.21}^{+0.18}$ \\
\hline WMAP9+BAO+Ly- $\alpha+$ Shear+Cluster $\left(B_{M}\right)$ & $0.289_{-0.08}^{+0.008}$ & $0.787_{-0.017}^{+0.019}$ & $0.16_{-0.09}^{+0.08}$ & $<0.30$ \\
\hline Planck & $0.355_{-0.061}^{+0.025}$ & $0.775_{-0.032}^{+0.077}$ & $<0.40$ & $<0.93$ \\
\hline Planck+Cluster & $0.272_{-0.018}^{+0.008}$ & $0.782_{-0.013}^{+0.027}$ & $<0.15$ & $<0.34$ \\
\hline Planck+Cluster $\left(B_{M}\right)$ & $0.287_{-0.015}^{+0.011}$ & $0.802_{-0.012}^{+0.025}$ & $<0.12$ & $<0.27$ \\
\hline Planck+Cluster $(\mathrm{BC})$ & $0.278_{-0.017}^{+0.009}$ & $0.790_{-0.013}^{+0.026}$ & $<0.14$ & $<0.32$ \\
\hline Planck+BAO & $0.309_{-0.009}^{+0.009}$ & $0.819_{-0.016}^{+0.027}$ & $<0.12$ & $<0.24$ \\
\hline Planck+Shear & $0.358_{-0.078}^{+0.051}$ & $0.708_{-0.082}^{+0.093}$ & $<0.78$ & $<1.12$ \\
\hline Planck + Ly- $\alpha$ & $0.329_{-0.024}^{+0.018}$ & $0.831_{-0.015}^{+0.024}$ & $<0.12$ & $<0.27$ \\
\hline Planck+BAO+Cluster & $0.300_{-0.010}^{+0.010}$ & $0.741_{-0.018}^{+0.015}$ & $0.32_{-0.07}^{+0.09}$ & $0.32_{-0.17}^{+0.17}$ \\
\hline Planck+BAO+Cluster $\left(B_{M}\right)$ & $0.300_{-0.009}^{+0.007}$ & $0.791_{-0.018}^{+0.020}$ & $0.15_{-0.08}^{+0.08}$ & $<0.28$ \\
\hline Planck $+\mathrm{BAO}+$ Shear & $0.306_{-0.011}^{+0.010}$ & $0.763_{-0.024}^{+0.025}$ & $0.26_{-0.10}^{+0.10}$ & $0.26_{-0.21}^{+0.20}$ \\
\hline $\mathrm{Planck}+\mathrm{BAO}+\mathrm{Ly}-\alpha$ & $0.310_{-0.008}^{+0.008}$ & $0.836_{-0.014}^{+0.016}$ & $<0.07$ & $<0.14$ \\
\hline Planck+BAO+Cluster $\left(B_{M}\right)+$ Shear & $0.300_{-0.011}^{+0.009}$ & $0.770_{-0.021}^{+0.021}$ & $0.22_{-0.10}^{+0.09}$ & $0.22_{-0.18}^{+0.17}$ \\
\hline Planck + BAO + Ly- $\alpha+$ Shear + Cluster $\left(B_{M}\right)$ & $0.293_{-0.010}^{+0.009}$ & $0.798_{-0.014}^{+0.018}$ & $<0.15$ & $<0.24$ \\
\hline $\operatorname{Planck}\left(A_{\mathrm{L}}\right)$ & $0.358_{-0.067}^{+0.040}$ & $0.716_{-0.066}^{+0.081}$ & $<0.70$ & $<1.17$ \\
\hline $\operatorname{Planck}\left(A_{\mathrm{L}}\right)+$ Cluster & $0.276_{-0.022}^{+0.010}$ & $0.769_{-0.018}^{+0.032}$ & $<0.22$ & $<0.41$ \\
\hline $\operatorname{Planck}\left(A_{\mathrm{L}}\right)+\mathrm{BAO}$ & $0.307_{-0.010}^{+0.010}$ & $0.778_{-0.033}^{+0.047}$ & $0.22_{-0.19}^{+0.09}$ & $<0.46$ \\
\hline $\operatorname{Planck}\left(A_{\mathrm{L}}\right)+\mathrm{BAO}+$ Cluster & $0.301_{-0.009}^{+0.009}$ & $0.734_{-0.015}^{+0.016}$ & $0.35_{-0.08}^{+0.08}$ & $0.35_{-0.16}^{+0.15}$ \\
\hline $\operatorname{Planck}\left(A_{\mathrm{L}}\right)+\mathrm{BAO}+$ Shear+ Cluster & $0.300_{-0.010}^{+0.009}$ & $0.733_{-0.014}^{+0.016}$ & $0.35_{-0.08}^{+0.08}$ & $0.35_{-0.16}^{+0.15}$ \\
\hline
\end{tabular}

\subsection{Massive neutrinos}

We first turn our attention to the degenerate active neutrino case, whose results are summarized in Table 1 and Fig. 1. To illustrate how different probes of the low redshift Universe combined with $\mathrm{CMB}$ measurements constrain cosmological parameters we use them one by one, before combining them together (see Fig. 2 and Fig. 3, respectively).

Combing with WMAP9 - The upper panels of Fig. 2 show constraints on the $\sigma_{8}-$ $\left(\sum m_{\nu}, \Omega_{\mathrm{m}}, H_{0}\right)$ planes and the 1D likelihood distribution of $\sum m_{\nu}$ for several datasets combined with WMAP9 as CMB data. None of them exhibit tension with WMAP9 results nor evidence for non-zero neutrino mass. The stronger constraint on the neutrino mass, 
$\sum m_{\nu}<0.29 \mathrm{eV}$, comes from the inclusion of Ly- $\alpha$ data, due to the high $\sigma_{8}$ value preferred by this dataset. Similar results (on $\sum m_{\nu}$ ) involve the inclusion of cluster data which shrinks and shifts the $\sigma_{8}-\Omega_{\mathrm{m}}$ contours toward lower values requiring small values for the total neutrino mass, $\sum m_{\nu}<0.34 \mathrm{eV}$. Repeating the analysis with a free mass bias parameter (Cluster $\left.\left(B_{M}\right)\right)$ or taking into account the baryon correction to the HMF (Cluster(BC)) slightly increases the $\sigma_{8}$ and $\Omega_{\mathrm{m}}$ values (see left panel of Fig 4) without significantly affecting the bound on $\sum m_{\nu}$ nor its best fit values. In the latter case the errors on $\sigma_{8}$ and $\Omega_{\mathrm{m}}$ remain unchanged while the suppression of the HMF with respect to the standard case causes the shift of the two parameters. Conversely, the inclusion of $B_{M}$ in the fit relaxes the bounds on $\sigma_{8}$ and $\Omega_{\mathrm{m}}$ and shifts their contours owing to the low value assumed by the bias, $B_{M} \sim 0.9$. On the other hand, BAO data shows a mild preference for larger neutrino mass (see right panel of Fig 2) which displaces the neutrino bounds to higher values, $\sum m_{\nu}<0.59 \mathrm{eV}$, reason for that being the tight constraints on $\Omega_{\mathrm{m}}$ and the low $\sigma_{8}$ value allowed by this datasets combination. Shear measurement, as cluster number counts, provides constraints on $\sigma_{8} \Omega_{\mathrm{m}}^{\gamma}$ but with a poorer constraining power than clusters data and with a degeneracy direction more similar to the one given by WMAP9 data; therefore the inclusion of this dataset entails only a small improvement on neutrino mass constraints.

We start now to perform joint analyses of different probes of the low redshift Universe. The results are presented in the upper panels of Fig 3. Both the additions of cluster and shear datasets to the WMAP9+BAO joint analysis result in a larger than $2 \sigma$ preference for massive neutrino yielding $\sum m_{\nu}=0.35 \pm 0.17 \mathrm{eV}$ and $\sum m_{\nu}=0.38_{-0.24}^{+0.23} \mathrm{eV}$, respectively. Also when the bias parameter is marginalized out the combination WMAP9+BAO+Cluster $\left(B_{M}\right)$ shows a $2 \sigma$ evidence for non-zero neutrino masses, although with larger error bars and a lower mean value: $\sum m_{\nu}=0.26 \pm 0.20 \mathrm{eV}$. The result can be understood as follows: the BAO scale measurements basically fix the matter density parameter thus breaking the $\sigma_{8}-\Omega_{\mathrm{m}}$ degeneracy typical of cluster and shear constraints. The tight constraints obtained for these two parameters along with the large value of $\Omega_{\mathrm{m}}$ (driven by the BAO data) and small value of $\sigma_{8}$ (driven by cluster abundance or shear measurements) are compensated with a large value of $\sum m_{\nu}$. At variance, Ly- $\alpha$ data prefers large value of the power spectrum normalization, and when joined with WMAP9+BAO data the large $\Omega_{\mathrm{m}}$ and $\sigma_{8}$ values inferred require small neutrino masses yielding the upper limit $\sum m_{\nu}<0.19 \mathrm{eV}$. The further inclusion of shear data in the WMAP9+BAO+Cluster $\left(B_{M}\right)$ analysis does not improve substantially the error on the neutrino mass, but decreases $\sigma_{8}$ by $\sim 0.01$ thus favouring slightly larger neutrino masses and increasing to $3 \sigma$ the significance of the mass detection.

Finally we jointly analyse the WMAP9, BAO, Ly- $\alpha$, shear and cluster data including $B_{M}$ into the fit obtaining $\sum m_{\nu}<0.30 \mathrm{eV}$. The addition of Ly- $\alpha$ forest measurements raises the power spectrum normalization by $\sim 0.04$ which causes a shift of the neutrino mass toward lower values and reduces to $1 \sigma$ the significance of the mass detection. We decided not to combine Ly- $\alpha$ and cluster data without marginalizing over the bias since the WMAP9+BAO+Ly- $\alpha$ and WMAP9+BAO+Cluster data are already in tension by more than $2 \sigma$ (see upper panels of Fig. 3).

Combining with Planck - Now we repeat the same analysis but replacing the WMAP9 dataset with Planck. The results are summarized in Table 1 and shown in the lower panels of Fig 2 and Fig 3. Planck provides not only tighter cosmological constraints than WMAP9 data but it also prefers a larger matter density parameters, which in turn lowers the derived $H_{0}$ value and increases the power spectrum normalization. For datasets which are consistent with Planck measurements, i.e. BAO and Ly- $\alpha$, the combination with this CMB data yields lower 

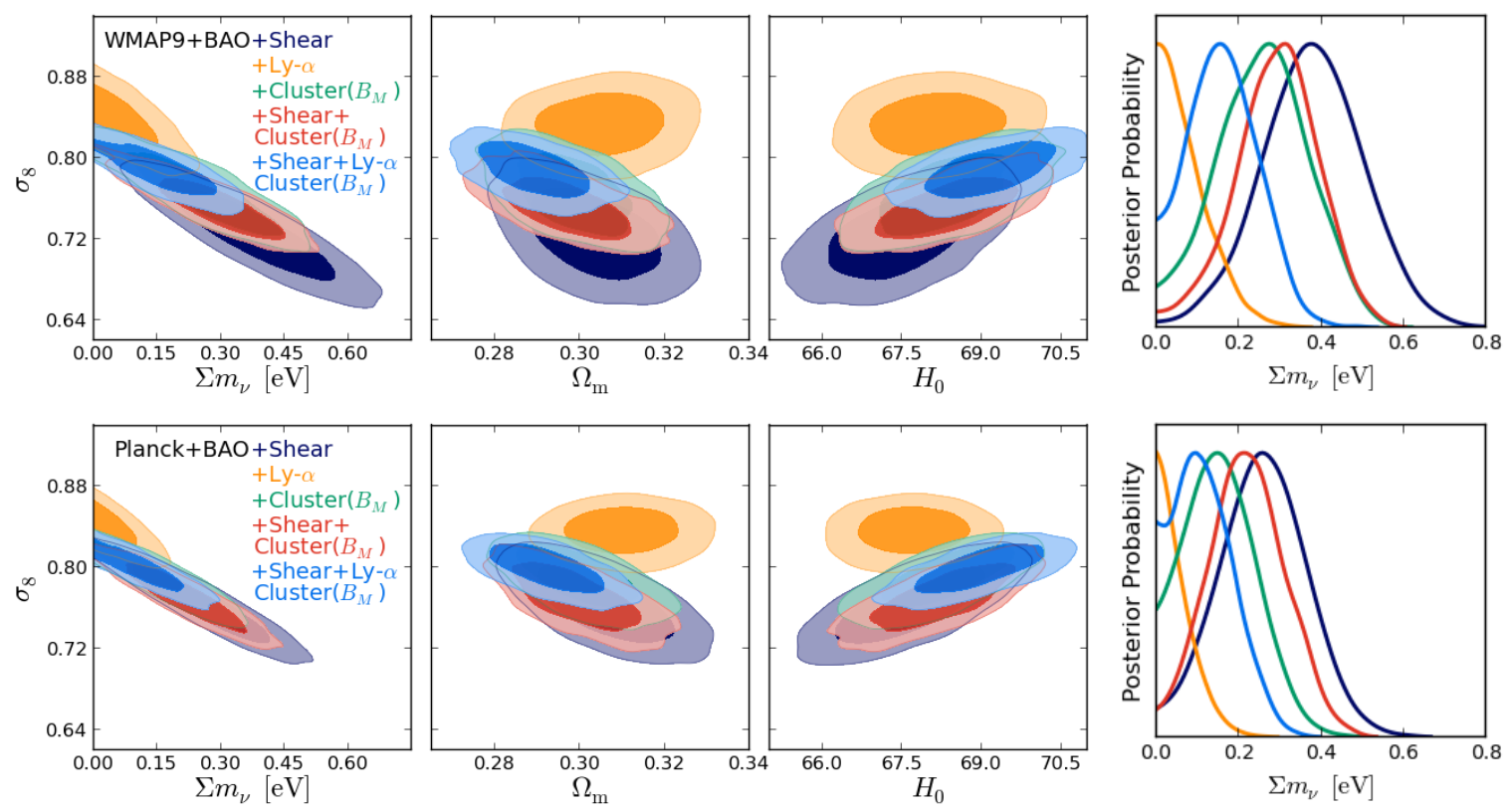

Figure 3: Left -Confidence contours at $68 \%$ and $95 \% \mathrm{CL}$ in the $\sigma_{8}-\left(\Omega_{\mathrm{m}}, H_{0}, \sum m_{\nu}\right)$ planes when combining WMAP9 (upper panels) or Planck (lower panels) with many different probes of the low redshift Universe within a $\Lambda \mathrm{CDM}+\sum m_{\nu}$ model. Right - Posterior probability distribution for $\sum m_{\nu}$ from the same datasets.

upper limits on the neutrino mass: $\sum m_{\nu}<0.27 \mathrm{eV}$ and $\sum m_{\nu}<0.24 \mathrm{eV}$ for Planck+Ly- $\alpha$ and Planck+BAO, respectively. In this case the $\mathrm{CMB}+\mathrm{BAO}$ combination does not show preference for large neutrino mass thanks to the larger value and tighter constraints on $\sigma_{8}$ provided by Planck. Conversely, the addition of shear or cluster data, which prefer lower $\sigma_{8}$, shifts the contours outside the region allowed by Planck by $1 \sigma$ and $2 \sigma$, respectively. This indicates that the extension to massive neutrino is not sufficient to bring the two datasets in agreement with Planck measurements. The shear measurements does not improve the constrains on $\sum m_{\nu}$, while clusters number counts yields an upper limit of $0.34 \mathrm{eV}$. Including in the cluster analysis the baryon correction to the HMF increases by few percents the $\sigma_{8}$ and $\Omega_{\mathrm{m}}$ values improving the fit by $\Delta \chi^{2} \simeq 2$, but it is not sufficient to relieve the tension between the two datasets. Allowing the bias to vary causes the contours to move towards the region allowed by Planck bringing the datasets in better agreement at the expense of a large mass bias, $B_{M} \sim 0.8$. In this case the best fit $\chi^{2}$ is reduced by $\sim 9$ with respect to the standard Planck+Cluster analysis and, as expected for consistent datasets, the errors shrink giving an upper limit of $\sum m_{\nu}<0.27 \mathrm{eV}$.

As above we start now to combine different probes of the low redshift Universe at the same time. The main results are shown in the lower panels of Fig. 3. Similar to the previous results the inclusion of cluster or shear datasets in the Planck $+\mathrm{BAO}$ joint analysis results in a preference for massive neutrinos at more than $2 \sigma$. We obtain $\sum m_{\nu}=0.32 \pm$ $0.17 \mathrm{eV}$ combining Planck, BAO and cluster data and $\sum m_{\nu}=0.26_{-0.21}^{+0.20} \mathrm{eV}$ replacing the latter with shear data. However, looking at the lower panels of Fig. 2 it is clear that the large mean value of $\sum m_{\nu}=0.32 \mathrm{eV}$ obtained from Planck+BAO+Cluster is driven by the tension between Planck+BAO and cluster constraints. In other words, the resulting 
constraints cannot be used to claim a significant detection of the neutrino mass, but rather they represent a compromise solution between discrepant datasets. Indeed, if we repeat the analysis marginalizing over the bias the best fit improves by $\Delta \chi^{2} \simeq 11$ - in this case the Planck+BAO and Planck+Cluster $\left(B_{M}\right)$ contours overlap (see middle panel of Fig. 4)- and we obtain only a mild preference for massive neutrino at $1 \sigma$ and an upper limit of $\sum m_{\nu}<0.28$ $\mathrm{eV}$ at $2 \sigma$. A very tight upper limit of $\sum m_{\nu}=0.14 \mathrm{eV}$ results instead from the combination of Planck with BAO and Ly- $\alpha$ data in agreement with the previous results obtained from WMAP9 data. Then we add progressively the shear and Ly- $\alpha$ constraints to the joint analysis Planck+BAO+Cluster $\left(B_{M}\right)$. Again the inclusion of shear measurements after cluster data does not alter significantly the error on $\sum m_{\nu}$, but lowers by $\sim 0.02$ the power spectrum normalization boosting the total neutrino mass to $\sum m_{\nu}=0.22_{-0.18}^{+0.17} \mathrm{eV}$, thus providing a $2 \sigma$ evidence for massive neutrinos. Instead, the shift to higher $\sigma_{8}$ value induced by the Ly- $\alpha$ dataset pushes again the mean neutrino mass toward lower value and wipes out the neutrino mass detection yielding $\sum m_{\nu}<0.24 \mathrm{eV}$.

Another possible way to relieve the tension between Planck and clusters data is to marginalize over the lensing contribution to the temperature power spectrum, parametrized by the parameter $A_{\mathrm{L}}$. The Planck Collaboration reported some anomalies when $A_{\mathrm{L}}$ is included in the fit: for a $\Lambda \mathrm{CDM}+A_{\mathrm{L}}$ model they found $A_{\mathrm{L}}=1.22_{-0.22}^{+0.25}$ [1], which is at $2 \sigma$ from the expected value of one and $1 \sigma$ away from the lensing signal extrapolated from the 4-point function $A_{\mathrm{L}}^{\phi \phi}=0.99_{-0.10}^{+0.11}$. Since Planck constraints on neutrino mass mainly relay on lensing information (massive neutrinos increase the expansion rate at $z \gtrsim 1$ suppressing clustering on sub-horizon scales at non-relativistic transition; see e.g. [1]) marginalizing over $A_{\mathrm{L}}$ significantly degrades the error on $\sum m_{\nu}$. Moreover, the preferred value of $A_{\mathrm{L}}>1$ shifts by $\sim 1 \sigma$ the $\Omega_{\mathrm{m}}-\sigma_{8}$ contours bringing Planck in much better agreement with cluster and shear data (see right panel of Fig. 4). The joint analysis Planck $\left(A_{\mathrm{L}}\right)+$ Cluster gives $\sum m_{\nu}<0.41$ $\mathrm{eV}$ with an improved best fit with respect to the Planck+Cluster analysis of $\Delta \chi^{2} \simeq 16$, while Planck $\left(A_{\mathrm{L}}\right)+\mathrm{BAO}$ yields $\sum m_{\nu}<0.46 \mathrm{eV}$ with a mild preference for massive neutrinos similar to the results obtained in combination with WMAP9. Combining Planck $\left(A_{\mathrm{L}}\right)$ with cluster and BAO yields $\sum m_{\nu}=0.35_{-0.16}^{+0.15} \mathrm{eV}$ : the tight constraints on $\sigma_{8}$ and $\Omega_{\mathrm{m}}$ provided by the combination of cluster and $\mathrm{BAO}$ data along with the low value of power spectrum normalization preferred by the former and large value of the matter density parameter preferred by the latter require large neutrino masses to bring the two datasets into agreement. The further inclusion of shear data, whose degeneracy direction between $\sigma_{8}$ and $\Omega_{\mathrm{m}}$ overlaps the one inferred from $\operatorname{Planck}\left(A_{\mathrm{L}}\right)$ data, does not change the parameter constraints nor shift their preferred values. For this analysis we do not consider the Ly- $\alpha$ dataset which exhibits a larger than $2 \sigma$ tension with $\operatorname{Planck}\left(A_{\mathrm{L}}\right)+$ Cluster and Planck $\left(A_{\mathrm{L}}\right)+$ shear results in the $\sigma_{8}-\Omega_{\mathrm{m}}$ plane due to the large value of the power spectrum normalization favoured by Ly- $\alpha$ forest data.

\subsection{Extra sterile massive neutrinos}

We now explore the scenario with an extra sterile neutrino component. Table 2 and Fig. 5 summarize the results obtained for the various data combinations. For this cosmological model we employ only Planck as CMB dataset since the constraints obtained from WMAP9 are much weaker than Planck ones. With the same logic adopted in the previous section we start combining single dataset with the CMB data and then we add them progressively. The inclusion of $N_{\text {eff }}$ in the fit opens new parameter degeneracies which relax the Planck contours and bring the cluster and shear constraints in better agreement with the CMB data. In 

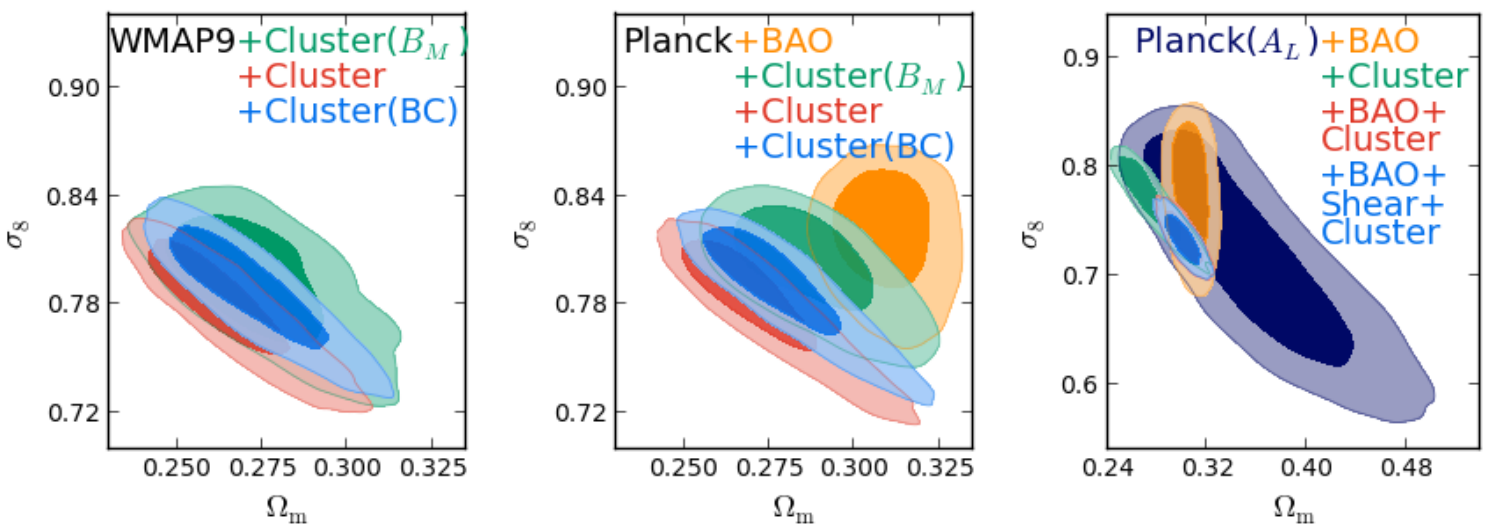

Figure 4: Left-Middle panel - Comparison of the confidence contours at $68 \%$ and $95 \%$ CL in the $\sigma_{8}-\Omega_{\mathrm{m}}$ plane within a $\Lambda \mathrm{CDM}+\sum m_{\nu}$ model when combining WMAP9 (left panel) or Planck (middle panel) with cluster data using different prescriptions: the standard one (Cluster $)$, the baryon correction $($ Cluster $(B C))$ or marginalizing over the bias $\left(C l u s t e r\left(B_{M}\right)\right)$. In the middle panel are also shown the confidence contours for the joint analysis Planck+BAO: only when $B_{M}$ is allowed to vary the Planck+BAO and Planck+Cluster $\left(B_{M}\right)$ regions overlap. Right panel - Joint $68 \%$ and $95 \%$ CL constraints on $\sigma_{8}-\Omega_{\mathrm{m}}$ for different dataset combined with Planck with the $A_{\mathrm{L}}$-lensing signal marginalized out.
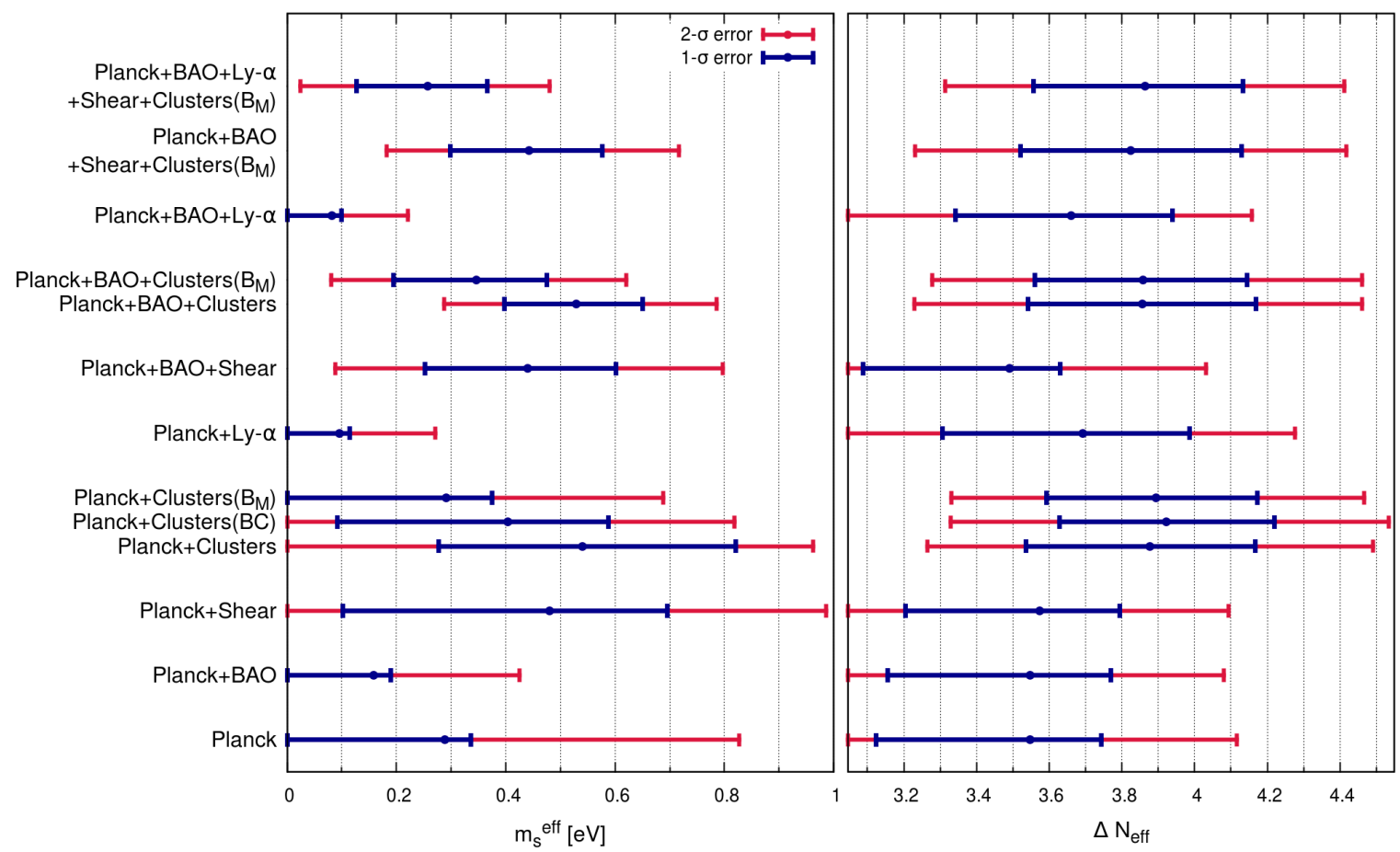

Figure 5: Summary of the $1 \sigma$ and $2 \sigma$ errors on $m_{\mathrm{s}}^{\text {eff }}$ and $N_{\text {eff }}$ obtained from the dataset combinations discussed in section 3.2 for a $\Lambda \mathrm{CDM}+m_{\mathrm{s}}^{\text {eff }}+N_{\text {eff }}$ model with one massive neutrino of mass $m_{\nu}=0.06 \mathrm{eV}$. 
Table 2: Constraints on $\Omega_{\mathrm{m}}, \sigma_{8}, m_{\mathrm{s}}^{\text {eff }}$ and $\Delta N_{\text {eff }}$ for a $\Lambda$ CDM model with massive sterile neutrino using different datasets. Errors are reported at $68 \%$ confidence level for $\sigma_{8}$ and $\Omega_{\mathrm{m}}$, and both $68 \%$ and $95 \%$ confidence level for $m_{\mathrm{s}}^{\text {eff }}$ and $\Delta N_{\text {eff. Notations included in parenthesis }}$ denote modifications to the standard setting: (BC) stands for the baryon correction to the HMF, while $\left(B_{M}\right)$ corresponds to analyses with the mass bias parameter marginalized out.

\begin{tabular}{|c|c|c|c|c|c|c|}
\hline \multirow[t]{2}{*}{ Dataset } & \multirow[t]{2}{*}{$\Omega_{\mathrm{m}}$} & \multirow[t]{2}{*}{$\sigma_{8}$} & \multicolumn{2}{|c|}{$m_{\mathrm{s}}^{\mathrm{eff}}[\mathrm{eV}]$} & \multicolumn{2}{|c|}{$\Delta N_{\text {eff }}$} \\
\hline & & & $68 \% \mathrm{CL}$ & $95 \% \mathrm{CL}$ & $68 \% \mathrm{CL}$ & $95 \% \mathrm{CL}$ \\
\hline Planck & $0.322_{-0.030}^{+0.025}$ & $0.800_{-0.031}^{+0.052}$ & $<0.34$ & $<0.86$ & $0.49_{-0.42}^{+0.18}$ & $<1.07$ \\
\hline Planck+Cluster & $0.304_{-0.027}^{+0.026}$ & $0.745_{-0.037}^{+0.023}$ & $0.54_{-0.26}^{+0.26}$ & $<0.98$ & $0.84_{-0.32}^{+0.32}$ & $0.84_{-0.60}^{+0.63}$ \\
\hline $\operatorname{Planck}+\operatorname{Cluster}\left(B_{M}\right)$ & $0.295_{-0.028}^{+0.019}$ & $0.794_{-0.032}^{+0.040}$ & $<0.38$ & $<0.69$ & $0.85_{-0.30}^{+0.28}$ & $0.85_{-0.57}^{+0.56}$ \\
\hline Planck+Cluster(BC) & $0.296_{-0.028}^{+0.023}$ & $0.770_{-0.036}^{+0.031}$ & $0.40_{-0.19}^{+0.31}$ & $<0.81$ & $0.88_{-0.29}^{+0.30}$ & $0.88_{-0.60}^{+0.61}$ \\
\hline Planck+BAO & $0.306_{-0.009}^{+0.009}$ & $0.818_{-0.026}^{+0.033}$ & $<0.19$ & $<0.43$ & $0.50_{-0.39}^{+0.22}$ & $<1.04$ \\
\hline Planck+Shear & $0.309_{-0.028}^{+0.028}$ & $0.752_{-0.043}^{+0.037}$ & $0.48_{-0.38}^{+0.22}$ & $<0.99$ & $0.53_{-0.37}^{+0.22}$ & $<1.30$ \\
\hline Planck + Ly- $\alpha$ & $0.309_{-0.024}^{+0.023}$ & $0.843_{-0.021}^{+0.021}$ & $<0.11$ & $<0.27$ & $0.65_{-0.38}^{+0.30}$ & $<1.49$ \\
\hline Planck+BAO+Cluster & $0.303_{-0.009}^{+0.009}$ & $0.744_{-0.014}^{+0.013}$ & $0.53_{-0.13}^{+0.12}$ & $0.53_{-0.24}^{+0.26}$ & $0.81_{-0.32}^{+0.31}$ & $0.81_{-0.63}^{+0.60}$ \\
\hline Planck+BAO+Cluster $\left(B_{M}\right)$ & $0.303_{-0.009}^{+0.007}$ & $0.782_{-0.018}^{+0.020}$ & $0.35_{-0.15}^{+0.13}$ & $0.35_{-0.27}^{+0.27}$ & $0.81_{-0.30}^{+0.29}$ & $0.81_{-0.58}^{+0.60}$ \\
\hline Planck+BAO+Shear & $0.305_{-0.010}^{+0.09}$ & $0.753_{-0.022}^{+0.023}$ & $0.44_{-0.19}^{+0.16}$ & $0.44_{-0.35}^{+0.34}$ & $0.45_{-0.40}^{+0.14}$ & $<0.99$ \\
\hline Planck $+\mathrm{BAO}+\mathrm{Ly}-\alpha$ & $0.305_{-0.010}^{+0.09}$ & $0.844_{-0.019}^{+0.020}$ & $<0.10$ & $<0.22$ & $0.61_{-0.32}^{+0.28}$ & $<1.11$ \\
\hline $\begin{array}{l}\text { Planck+BAO+ } \\
\text { Shear+Cluster }\left(B_{M}\right)\end{array}$ & $0.303_{-0.009}^{+0.009}$ & $0.759_{-0.020}^{+0.017}$ & $0.44_{-0.14}^{+0.14}$ & $0.44_{-0.26}^{+0.28}$ & $0.78_{-0.30}^{+0.31}$ & $0.78_{-0.59}^{+0.60}$ \\
\hline $\begin{array}{l}\text { Planck+BAO+Ly- } \alpha \\
\text { Shear+Cluster }\left(B_{M}\right)\end{array}$ & $0.293_{-0.008}^{+0.009}$ & $0.794_{-0.016}^{+0.016}$ & $0.26_{-0.13}^{+0.11}$ & $0.26_{-0.24}^{+0.22}$ & $0.82_{-0.31}^{+0.27}$ & $0.82_{-0.55}^{+0.55}$ \\
\hline
\end{tabular}

particular, $N_{\text {eff }}>3.046$ increases the radiation energy content (see Eq. 2.2) and affects the expansion rate of the Universe thus relaxing the bounds on $H_{0}$ and the scalar spectral index, with which $N_{\text {eff }}$ is positively correlated. When cluster constraints are included we find a mild preference for massive sterile neutrino $m_{\mathrm{s}}^{\text {eff }}=0.54 \pm 0.26 \mathrm{eV}(68 \%)$, and a $2 \sigma$ hint for extra dark radiation $\Delta N_{\text {eff }}=0.84_{-0.60}^{+0.63}$. At variance with the Planck+Cluster joint analysis performed in the previous section - i.e. within a $\Lambda \mathrm{CDM}+\sum m_{\nu}$ model - this time the Planck+Cluster combination does not exhibit strong tension with the Planck results, which improves the $\chi^{2}$ best fit by $\simeq 11$ (e.g. compare the $\sigma_{8}-\Omega_{\mathrm{m}}$ panels of Fig. 2 and Fig. 6). This fact, along with the lower $\sigma_{8}$ and larger $H_{0}-n_{s}$ values preferred by cluster data and the positive correlation between $m_{\mathrm{s}}^{\text {eff }}$ and $\Delta N_{\text {eff }}$ accounts for the shift of the two parameters with respect to the Planck-only analysis (see Fig. 6). Analogous constraints on $m_{\mathrm{s}}^{\text {eff }}$ are provided by the inclusion of shear measurements which lowers the $\sigma_{8}$ mean value, while leaving the bounds on $n_{s}$ and $H_{0}$, and thus on $\Delta N_{\text {eff }}$, unaffected with respect to the Planck-only results. If we consider the Planck+Cluster analysis, the BC to the HMF results in a $1 \sigma$ shift of the power spectrum normalization toward higher values which reduces the $m_{\mathrm{s}}^{\text {eff }}$ mean value, while keeping the constraints on $N_{\text {eff }}$ and the best fit value almost unchanged (see Fig. 7). Similarly, but with an increased magnitude, if we repeat the analysis marginalizing over the bias the preferred $\sigma_{8}$ value shifts by $2 \sigma$ at the expense of a large value for the bias, $B_{M} \sim 0.8$, wiping out the former $1 \sigma$ preference for $m_{\mathrm{s}}^{\mathrm{eff}}$ larger than zero. For this analysis we find a mild improvement of the best fit value of $\Delta \chi^{2} \simeq 5$ with respect to the standard one. At odds the inclusion of BAO data reduces the error on $\Omega_{\mathrm{m}}$ and slightly increases the $\sigma_{8}$ mean value with respect to the Planck-only analysis. This results in tighter constraints for the sterile neutrino mass, $m_{\mathrm{s}}^{\mathrm{eff}}<0.43 \mathrm{eV}$, and leaves almost unchanged the bounds on $\Delta N_{\text {eff }}$. When joined to the Planck analysis the Ly- $\alpha$ data constrains $\sigma_{8}$ in the high values region allowed 



Figure 6: Two dimensional likelihood contours at $68 \%$ and $95 \%$ CL for $\sigma_{8}-\left(\Omega_{\mathrm{m}}, m_{\mathrm{s}}^{\text {eff }}\right)$ and $N_{\text {eff }}-m_{\mathrm{s}}^{\text {eff }}$ from Planck combined with different probes of the low redshift Universe within a $\Lambda \mathrm{CDM}+m_{\mathrm{s}}^{\text {eff }}+\Delta N_{\text {eff }}$ model. The dashed line shown in the $N_{\text {eff }}-m_{\mathrm{s}}^{\text {eff }}$ plane represent the prior on the physical sterile neutrino mass: $m_{\mathrm{s}}^{\text {eff }} /\left(\Delta N_{\mathrm{eff}}\right)^{3 / 4}<10 \mathrm{eV}$.
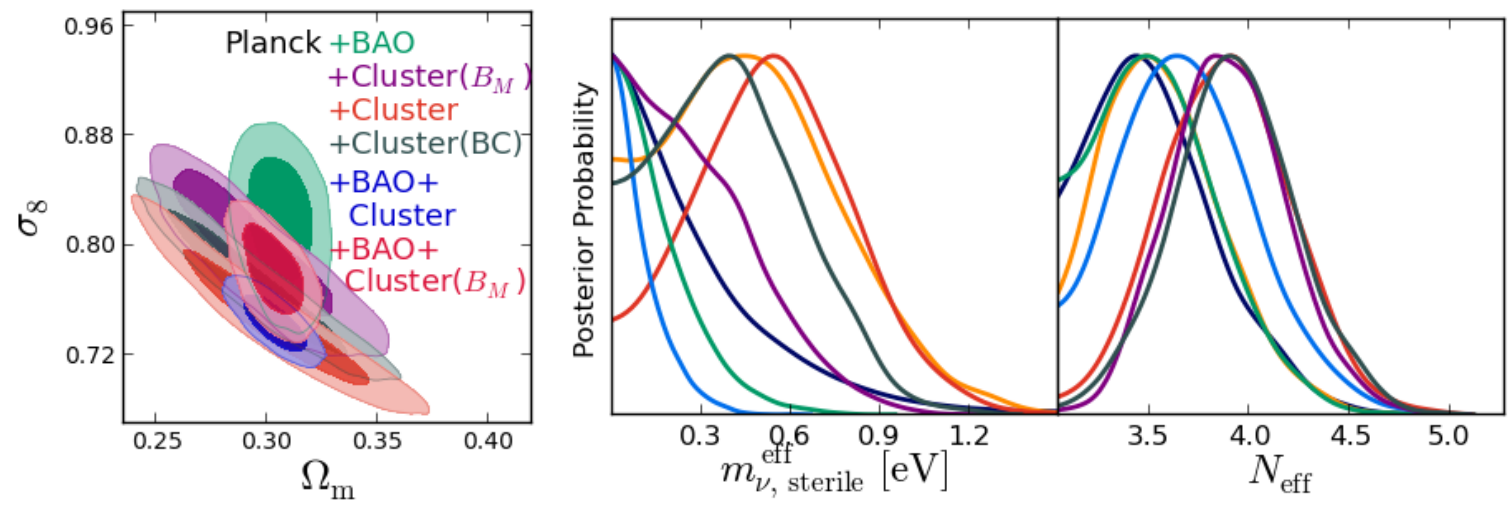

Figure 7: Left panel - Comparison of the two dimensional likelihood contours at $68 \%$ and $95 \% \mathrm{CL}$ in the $\sigma_{8}-\Omega_{\mathrm{m}}$ plane from the joint analysis of Planck or Planck+BAO and cluster data using different prescriptions for the HMF: the standard one (Cluster), the baryon correction $($ Cluster $(B C))$ or marginalizing over the bias $\left(\right.$ Cluster $\left.\left(B_{M}\right)\right)$. Right panels - Posterior probability distributions for $m_{\mathrm{s}}^{\text {eff }}$ and $N_{\text {eff }}$ from single datasets combined with Planck within a $\Lambda \mathrm{CDM}+m_{\mathrm{s}}^{\text {eff }}+\Delta N_{\text {eff }}$ model. The colour coding of the lines is the same of Fig. 6 and the left panel of this figure.

by Planck data and slightly increases the $n_{\mathrm{s}}$ and $H_{0}$ mean values. This entails an upper limit of $0.27 \mathrm{eV}$ for the effective sterile neutrino mass and a $\sim 20 \%$ increase of the $\Delta N_{\text {eff }}$ mean values.

We start now to combine Planck CMB measurements with different low redshift probes. The main results of these analyses are shown in Fig. 8. Also shown in the $N_{\text {eff }}-m_{\mathrm{s}}^{\text {eff }}$ plane are the $m_{\mathrm{s}}^{\text {eff }}$ values motivated by reactor and Gallium anomalies $\left(m_{\mathrm{s}}^{\text {eff }} \sim 1 \mathrm{eV}\right)$ and accelerator anomaly $\left(m_{\mathrm{s}}^{\mathrm{eff}} \sim 0.70 \mathrm{eV}\right)$, for a fully-thermalised sterile neutrino component $\left(\Delta N_{\text {eff }}=1\right)$. As for the $\Lambda \mathrm{CDM}+\sum m_{\nu}$ model, including BAO data in the Planck+Cluster or Planck+Shear analyses provides a $2 \sigma$ evidence for massive sterile neutrino $-m_{\mathrm{s}}^{\text {eff }}=0.53_{-0.24}^{+0.26}$ $\mathrm{eV}$ and $m_{\mathrm{s}}^{\text {eff }}=0.44_{-0.35}^{+0.34} \mathrm{eV}$, respectively - reason for that being the tight constraints on 

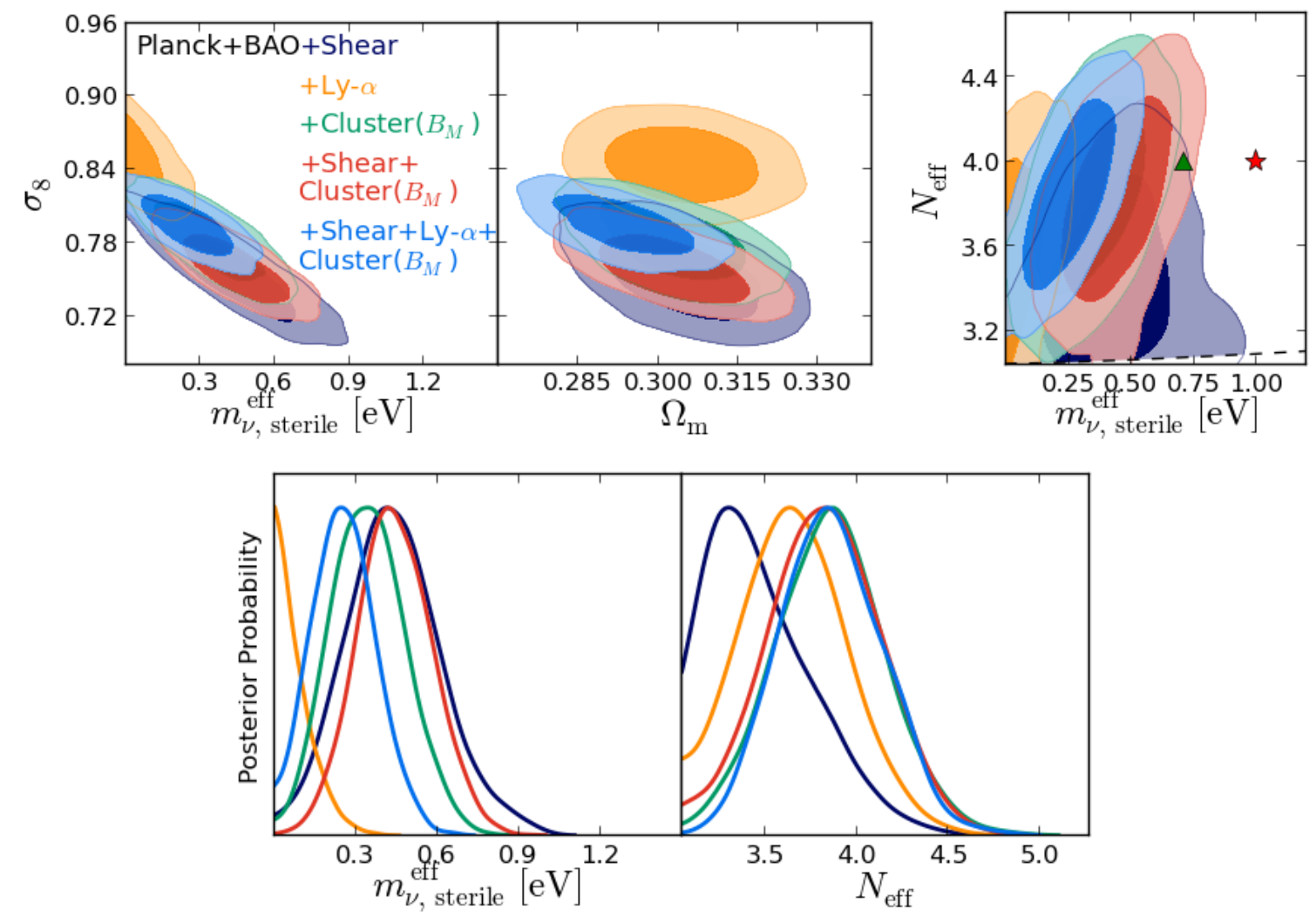

Figure 8: Upper panels - Confidence contours at $68 \%$ and $95 \%$ CL in the $\sigma_{8}-\left(\Omega_{\mathrm{m}}, m_{\mathrm{s}}^{\text {eff }}\right)$ and $N_{\text {eff }}-m_{\mathrm{s}}^{\text {eff }}$ planes from Planck combined with various probes of the low redshift Universe within a $\Lambda \mathrm{CDM}+m_{\mathrm{s}}^{\text {eff }}+\Delta N_{\text {eff }}$ model. Also shown in the $N_{\text {eff }}-m_{\mathrm{s}}^{\text {eff }}$ plane the value of the effective sterile neutrino masses suggested by accelerator (green triangle) and reactor and Gallium (red star) anomalies assuming $\Delta N_{\text {eff }}=1$. Lower panels - 1D likelihood distributions for $m_{\mathrm{s}}^{\text {eff }}$ and $N_{\text {eff }}$ for the same datasets combinations.

$\Omega_{\mathrm{m}}$ from BAO measurements, which allow to break the $\sigma_{8}-\Omega_{\mathrm{m}}$ degeneracy, and the low $\sigma_{8}$ mean value preferred by cluster and shear data. As for $\Delta N_{\text {eff }}$ the Planck+BAO+Shear combination shows only a mild preference for $\Delta N_{\text {eff }}>0$, while the Planck $+\mathrm{BAO}+$ Cluster joint analysis, driven by the large $n_{\mathrm{S}}$ value required by cluster data, favours at $2 \sigma$ an extra dark radiation component. However, even if not as strong as for the $\Lambda \mathrm{CDM}+\sum m_{\nu}$ model, the Planck+BAO and Planck+Cluster results still exhibits a $\sim 1 \sigma$ tension (see right panel of Fig. 7), which could drive the large $m_{\mathrm{s}}^{\text {eff }}$ value obtained from the combination of the three datasets. Interestingly, at variance with the $\Lambda \mathrm{CDM}+\sum m_{\nu}$ analysis, the $2 \sigma$ detection of massive sterile neutrino remains also if we repeat the Planck+BAO+Cluster analysis including the uncertainty in the mass bias. In this case Planck, BAO and $\operatorname{cluster}\left(B_{M}\right)$ results are in good agreement and the inclusion of cluster data in the Planck+BAO joint analysis does not shift the contours outside the region allowed by the latter but only shrinks the errors, as expected for consistent datasets. Compared to the previous analysis without mass bias the fit improves by $\Delta \chi^{2} \simeq 5$. The inclusion of $B_{M}$ in the fit increases the $\sigma_{8}$ mean value by $\sim 0.04$ which involves a decrease of $\sim 35 \%$ of the mean effective sterile neutrino mass, $m_{\mathrm{s}}^{\text {eff }}=0.35 \pm 0.27 \mathrm{eV}$, while leaving unaffected the bounds on $\Delta N_{\text {eff. }}$ This datasets 
combination rejects at more than $3 \sigma$ the vanilla $\Lambda$ CDM model, which is located at the origin of $N_{\text {eff }}-m_{\mathrm{s}}^{\text {eff }}$ plane in Fig. 8, but it also disfavours a sterile neutrino mass of $m_{\mathrm{s}}^{\text {eff }} \sim 0.70 \mathrm{eV}$ as suggested by accelerator experiments, and even more strongly rejects the value of $m_{\mathrm{s}}^{\text {eff }} \sim 1 \mathrm{eV}$ motivated by reactor and Gallium experiments. The further inclusion of shear measurements has the effect of favour a slightly lower value of $\sigma_{8}$, which increases the mean sterile neutrino mass by $30 \%$ with respect to the Planck+BAO+Cluster $\left(B_{M}\right)$ analysis, thus strengthening the evidence for a light sterile neutrino species. Moreover, this shift is sufficient to bring the $N_{\text {eff }}-m_{\mathrm{s}}^{\text {eff }}$ confidence contours in agreement with the sterile neutrino mass motivated by accelerator experiments within $2 \sigma$. As in the massive active neutrino scenario, the inclusion of Ly- $\alpha$ data on the Planck+BAO analysis leads to opposite results. The large value of $\sigma_{8}$ preferred by Ly- $\alpha$ data requires small value for the sterile neutrino mass to be consistent with the Planck+BAO constraints. This provides a thigh upper limit of $0.22 \mathrm{eV}$ on $m_{\mathrm{s}}^{\text {eff }}$ in agreement with the standard $\Lambda \mathrm{CDM}$ predictions. Finally, we add the Ly- $\alpha$ datasets to the combination Planck+BAO+Shear+Cluster $\left(B_{M}\right)$ finding $m_{\mathrm{s}}^{\mathrm{eff}}=0.26_{-0.24}^{+0.22} \mathrm{eV}$. The Ly- $\alpha$ contours are in tension with the Planck+BAO+Shear+Cluster $\left(B_{M}\right)$ results at more than $1 \sigma$, and these constraints on $m_{\mathrm{s}}^{\text {eff }}$ reflect the compromise between the low $\sigma_{8}$ mean value preferred by shear and cluster data and the large one inferred from Ly- $\alpha$ data. As for $\Delta N_{\text {eff }}$, the inclusion of the Ly- $\alpha$ data does not shift its mean value but helps to reduce the errors giving $\Delta N_{\text {eff }}=0.82 \pm 0.55$, that is a larger than $2 \sigma$ preference for extra dark radiation.

\section{Conclusions}

Many recent studies investigated extensions of the $\Lambda$ CDM model that include massive neutrinos as a possible means to relieve the tension between Planck CMB data and several measurements of the large scale structure (LSS), finding preferences for non-vanishing neutrino mass. The neutrino mass claimed detections are mainly driven by low redshift growth of structure constraints, and in particular by galaxy clusters data, thanks to the tight constraints that they provide on the combination $\sigma_{8}\left(\Omega_{\mathrm{m}} / 0.27\right)^{\gamma}$. However, neutrino constraints from cluster data suffer from systematic errors manly related to the uncertainty in the bias of the mass-observable relation and the calibration of the HMF in cosmology with massive neutrino, which often are not taken into account in data analysis. Here we presented neutrino mass constraints, either for an active and sterile neutrino scenario, from the combination of CMB measurements with low redshift Universe probes. We employed for our analysis CMB measurements from WMAP9 or Planck in combination with BAO scale measurements from BOSS DR11, galaxy shear power spectrum from CFTHLenS, SDSS Ly- $\alpha$ forest power spectrum constraints and cluster mass functions from Chandra observations. At variance with previous similar studies, which included in their analysis constraints derived within a vanilla $\Lambda$ CDM model, we performed a full likelihood analysis for all the datasets employed in this work in order to avoid model dependence of the constraints. Moreover, in the cluster data analysis we properly take into account the impact of massive neutrino in the HMF calibration and we investigated the effects on cosmological constraints of the uncertainty in the mass bias and re-calibration of the HMF due to baryonic feedback processes as suggested in [41].

For both neutrino scenarios assumed and CMB datasets employed, we found that none of the constraints from the LSS data, combined on a one-by-one basis, with CMB measurements provide strong - i.e. larger than $2 \sigma$ - evidence for massive neutrino. From the joint analysis Planck+Cluster we obtained $\sum m_{\nu}<0.34 \mathrm{eV}$ but we emphasize that the extensions to three massive active neutrinos is not sufficient to bring the dataset in agreement 
with Planck results. Indeed the extension to massive neutrinos does not improve the fit of the combination of Planck and galaxy cluster data, with respect to the vanilla $\Lambda$ CDM model. Taking into account the effect of baryons on the HMF calibration or the uncertainty in the mass bias increases the $\sigma_{8}$ mean value improving the fit with respect to the standard analysis of $\Delta \chi^{2} \simeq 2$ and $\Delta \chi^{2} \simeq 9$, respectively. In the latter case constraints from Planck CMB and galaxy clusters agree within $1 \sigma$, with their combination preferring a vanishing neutrino mass. Alternatively, the Planck and cluster datasets can be brought in agreement marginalizing over the lensing contribution to the temperature power spectrum. In this case the Planck' s $\sigma_{8}-\Omega_{\mathrm{m}}$ contours relax and shift by $\sim 1 \sigma$, improving the $\chi^{2}$ best fit value by $\sim 16$ with respect to the Planck+Cluster analysis. Similarly, assuming an extra sterile neutrino species, which introduces in the fit the additional parameter $\Delta N_{\text {eff }}$, relaxes the Planck's bounds reducing the discrepancy with cluster results by $\Delta \chi^{2} \simeq 11$ with respect to the same data combination in a $\Lambda \mathrm{CDM}+\sum m_{\nu}$ model. From this analysis we obtained a $1 \sigma$ preference for non vanishing neutrino mass and $\Delta N_{\text {eff }}=0.84_{-0.60}^{+0.63}$. Including also the mass bias in the fit further improves the agreement between Planck and cluster datasets by $\Delta \chi^{2} \simeq 5$ at the expense of an higher $\sigma_{8}$ mean value which cancels the former $1 \sigma$ detection of massive neutrinos. Preference for non-vanishing neutrino mass at more than $2 \sigma$ were found instead combining $\mathrm{CMB}$ and $\mathrm{BAO}$ measurements with shear or cluster data. The BAO constraints break the $\sigma_{8}-\Omega_{\mathrm{m}}$ degeneracy typical of cluster and shear data while the low $\sigma_{8}$ mean value preferred by the latter is compensated by large neutrino mass. However, for the $\Lambda \mathrm{CDM}+\sum m_{\nu}$ model, the large neutrino mass obtained from the joint analysis Planck $+\mathrm{BAO}+\mathrm{Cluster}$ is driven by the tension between Planck+BAO and cluster constraints. Indeed, including the mass bias parameter in the fit reduces the $\sum m_{\nu}$ mean value by $50 \%$ wiping out the $2 \sigma$ preference for massive neutrino, but increasing the $\chi^{2}$ best fit value by $\sim 11$. For the sterile neutrino case, when considering the uncertainty in the mass bias, the fit is improved by $\Delta \chi^{2} \simeq 5$ at the expenses of a lower mean neutrino mass but still with a preference for an extra massive neutrino, $m_{\mathrm{s}}^{\text {eff }}=0.35 \pm 0.27 \mathrm{eV}-\Delta N_{\text {eff }}=0.81_{-0.58}^{+0.60}$. The significance of the detection increases further including simultaneously shear and cluster data. For a $\Lambda$ CDM model with three degenerate massive neutrinos we obtained $\sum m_{\nu}=0.29_{-0.21}^{+0.18} \mathrm{eV}$ from the combination WMAP9+BAO+Shear+Cluster $\left(B_{M}\right)$, while replacing WMAP9 with Planck measurements we got $\sum m_{\nu}=0.22_{-0.18}^{+0.17} \mathrm{eV}$, or $\sum m_{\nu}=0.35_{-0.16}^{+0.15} \mathrm{eV}$ marginalizing over the lensing signal. For the sterile neutrino case, from the combination Planck+BAO+Shear+Cluster $\left(B_{M}\right)$, we found $m_{\mathrm{s}}^{\mathrm{eff}}=0.44_{-0.26}^{+0.28} \mathrm{eV}$ and $\Delta N_{\mathrm{eff}}=0.78_{-0.59}^{+0.60}$, that is a larger than $3 \sigma$ rejection of the vanilla $\Lambda \mathrm{CDM}$ model. Assuming a fully thermalised sterile neutrino these constraints reject at even higher significance a $1.0 \mathrm{eV}$ sterile neutrino as motived by reactor and Gallium anomalies, while a neutrino mass of $0.7 \mathrm{eV}$ as suggested by accelerator anomaly is within the $2 \sigma$ errors. Conversely, the Ly- $\alpha$ measurements tend to increase the $\sigma_{8}$ mean value with respect to the CMB data analyses, which in turn entails a preference for vanishing neutrino masses to be consistent with the other parameters constraints. For the active neutrino scenario we got $\sum m_{\nu}<0.19 \mathrm{eV}$ and $\sum m_{\nu}<0.14 \mathrm{eV}$ combining BAO, Ly- $\alpha$ and WMAP9 or Planck dataset, respectively. Similarly, for the sterile neutrino model we obtained $m_{\mathrm{s}}^{\text {eff }}<0.22 \mathrm{eV}$ and $\Delta N_{\text {eff }}<1.11$. The full data combination provides neutrino mass constraints which reflects the compromise between the $\sigma_{8}$ values preferred by shear and cluster data and those inferred from Ly- $\alpha$ measurements. For the $\Lambda \mathrm{CDM}+\sum m_{\nu}$ model we obtained only an upper limit on the total neutrino mass independently from the CMB dataset employed, while in the sterile neutrino scenario we still found a $2 \sigma$ preference for an extra massive species, $m_{\mathrm{s}}^{\mathrm{eff}}=0.26_{-0.24}^{+0.22} \mathrm{eV}-\Delta N_{\mathrm{eff}}=0.82 \pm 0.55$. 
In summary, our results highlight that current CMB and LSS probes point towards a significant detection of the sterile neutrino mass and dark radiation unless the constraints on $\sigma_{8}$ provided by clusters and shear data turn out to be biased toward lower values. As for clusters, this could be due to a possible underestimate of the cluster mass bias. As for cosmic shear, an underestimate of $\sigma_{8}$ could be induced by a misinterpretation of the intrinsic alignment signal. On the other hand new BOSS results from the $1 \mathrm{D}$ Ly- $\alpha$ flux power spectrum or Planck CMB data - e.g. due to a different foreground removal technique [88] - could strengthen or weaken the evidence for non-vanishing neutrino masses.

More in general, our results highlight that current cosmological data already have the potential to set rather stringent constraints on neutrino masses, which could even challenge the results from laboratory experiments, but these are hampered by systematics which need to be better controlled and understood. This becomes even more important in view of future surveys (eROSITA, SPT3G, DES, DESI, Euclid), that thanks to the large amount of data to be provided will bring down the statistical errors by large factors. As for cluster cosmology, the ever increasing number of high quality weak lensing data is expected to provide in the near term well characterized and unbiased constraints on the absolute cluster mass calibration. From the theoretical side, refined cosmological simulations which properly accounts for neutrino and baryonic physics will be crucial to improve the calibration of the HMF and modelling of the shear and Ly- $\alpha$ forest flux power spectrum.

\section{Acknowledgments}

We thank Martin Kilbinger and Alexey Vikhlinin for useful discussions. This work has been supported by the PRIN-MIUR 201278X4FL grant, by the IS PD51 INDARK INFN grant and by "Consorzio per la Fisica di Trieste". MV is supported by the ERC Starting Grant CosmoIGM.

\section{References}

[1] Planck Collaboration, Planck 2013 results. XVI. Cosmological parameters, ArXiv e-prints [arXiv: 1303.5076].

[2] A. G. Riess, L. Macri, S. Casertano, H. Lampeitl, H. C. Ferguson, A. V. Filippenko, S. W. Jha, W. Li, and R. Chornock, A 3\% Solution: Determination of the Hubble Constant with the Hubble Space Telescope and Wide Field Camera 3, Astrophysical Journal 730 119, [arXiv:1103.2976].

[3] C. Heymans, E. Grocutt, A. Heavens, and et al., CFHTLenS tomographic weak lensing cosmological parameter constraints: Mitigating the impact of intrinsic galaxy alignments, Montly Notices of the Royal Astronomical Society 432 2433-2453, [arXiv:1303.1808].

[4] R. Mandelbaum, A. Slosar, T. Baldauf, and et al, Cosmological parameter constraints from galaxy-galaxy lensing and galaxy clustering with the SDSS DR7, Montly Notices of the Royal Astronomical Society 432 1544-1575, [arXiv: 1207.1120].

[5] F. Beutler, S. Saito, H.-J. Seo, and et al., The clustering of galaxies in the SDSS-III Baryon Oscillation Spectroscopic Survey: Testing gravity with redshift-space distortions using the power spectrum multipoles, ArXiv e-prints [arXiv:1312.4611].

Note added: during the finalization of this work, Ref [89] presented constrains on $\sum m_{\nu}$ from the combination of WMAP9 or Planck CMB data with a sample of 224 cluster from the ROSAT All-Sky survey clusters with masses calibrated using weak lensing data from Subaru and CFHT telescopes for 50 galaxy clusters. Even if their results are not directly comparable with ours they are qualitatively in agreement. 
[6] A. Vikhlinin, A. V. Kravtsov, R. A. Burenin, H. Ebeling, W. R. Forman, and et al., Chandra Cluster Cosmology Project III: Cosmological Parameter Constraints, Astrophysical Journal 692 1060-1074, [arXiv: 0812.2720].

[7] E. Rozo, R. H. Wechsler, E. S. Rykoff, and et al., Cosmological Constraints from the Sloan Digital Sky Survey maxBCG Cluster Catalog, Astrophysical Journal 708 645-660, [arXiv:0902.3702].

[8] S. W. Allen, A. E. Evrard, and A. B. Mantz, Cosmological Parameters from Observations of Galaxy Clusters, Annual Review of Astronomy \&3 Astrophysics 49 409-470, [arXiv: 1103.4829].

[9] Planck Collaboration, P. A. R. Ade, N. Aghanim, C. Armitage-Caplan, M. Arnaud, M. Ashdown, F. Atrio-Barandela, J. Aumont, C. Baccigalupi, A. J. Banday, and et al., Planck 2013 results. XX. Cosmology from Sunyaev-Zeldovich cluster counts, ArXiv e-prints [arXiv: 1303.5080].

[10] J. Lesgourgues and S. Pastor, Massive neutrinos and cosmology, Physics Reports 429 307-379, [astro-ph/].

[11] Y. Y. Y. Wong, Neutrino Mass in Cosmology: Status and Prospects, Annual Review of Nuclear and Particle Science 61 69-98, [arXiv:1111.1436].

[12] J. Lesgourgues, G. Mangano, G. Miele, and S. Pastor, Neutrino Cosmology. Feb., 2013.

[13] Particle Data Group Collaboration, J. Beringer, J. F. Arguin, and Barnett, Beringer, j. and et al. (particle data group), Phys. Rev. D 86010001.

[14] M. C. Gonzalez-Garcia, M. Maltoni, J. Salvado, and T. Schwetz, Global fit to three neutrino mixing: critical look at present precision, Journal of High Energy Physics 12 123, [arXiv: 1209.3023].

[15] J. M. Conrad, W. C. Louis, and M. H. Shaevitz, The LSND and MiniBooNE Oscillation Searches at High $\Delta m^{2}$, Annual Review of Nuclear and Particle Science 63 45-67, [arXiv: 1306.6494].

[16] K. N. Abazajian, M. A. Acero, S. K. Agarwalla, and et al., Light Sterile Neutrinos: A White Paper, ArXiv e-prints [arXiv:1204.5379].

[17] R. A. Burenin, Possible indication for non-zero neutrino mass and additional neutrino species from cosmological observations, Astronomy Letters 39 357-366, [arXiv:1301.4791].

[18] M. Wyman, D. H. Rudd, R. A. Vanderveld, and W. Hu, nu-LCDM: Neutrinos reconcile Planck with the Local Universe, ArXiv e-prints [arXiv:1307.7715].

[19] R. A. Battye and A. Moss, Evidence for massive neutrinos from CMB and lensing observations, ArXiv e-prints [arXiv:1308.5870].

[20] J. Hamann and J. Hasenkamp, A new life for sterile neutrinos: resolving inconsistencies using hot dark matter, ArXiv e-prints [arXiv:1308.3255].

[21] M. Archidiacono, N. Fornengo, C. Giunti, S. Hannestad, and A. Melchiorri, Sterile neutrinos: Cosmology versus short-baseline experiments, Physical Review D 87 125034, [arXiv: 1302.6720].

[22] F. Beutler, S. Saito, J. R. Brownstein, and et al., The clustering of galaxies in the SDSS-III Baryon Oscillation Spectroscopic Survey: Signs of neutrino mass in current cosmological datasets, ArXiv e-prints [arXiv:1403.4599].

[23] E. Giusarma, E. Di Valentino, M. Lattanzi, A. Melchiorri, and O. Mena, Relic Neutrinos, thermal axions and cosmology in early 2014, ArXiv e-prints [arXiv:1403.4852].

[24] M. Archidiacono, N. Fornengo, S. Gariazzo, C. Giunti, S. Hannestad, and M. Laveder, Light sterile neutrinos after BICEP-2, Journal of Cosmology and Astroparticle Physics 6 31, [arXiv: 1404.1794]. 
[25] S. M. Feeney, H. V. Peiris, and L. Verde, Is there evidence for additional neutrino species from cosmology?, Journal of Cosmology and Astroparticle Physics 4 36, [arXiv:1302.0014].

[26] L. Verde, S. M. Feeney, D. J. Mortlock, and H. V. Peiris, (Lack of) Cosmological evidence for dark radiation after Planck, Journal of Cosmology and Astroparticle Physics 9 13, [arXiv: 1307.2904].

[27] B. Leistedt, H. V. Peiris, and L. Verde, No new cosmological concordance with massive sterile neutrinos, ArXiv e-prints [arXiv: 1404.5950].

[28] A. V. Kravtsov and S. Borgani, Formation of Galaxy Clusters, Annual Review of Astronomy \&3 Astrophysics 50 353-409, [arXiv: 1205.5556].

[29] A. Mantz, S. W. Allen, D. Rapetti, and H. Ebeling, The observed growth of massive galaxy clusters - I. Statistical methods and cosmological constraints, Montly Notices of the Royal Astronomical Society 406 1759-1772, [arXiv:0909.3098].

[30] B. A. Benson, T. de Haan, J. P. Dudley, C. L. Reichardt, Aird, and et al., Cosmological Constraints from Sunyaev-Zel'dovich-selected Clusters with X-Ray Observations in the First 178 $d^{d e g^{2}}$ of the South Pole Telescope Survey, Astrophysical Journal 763 147, [arXiv:1112.5435].

[31] M. Hasselfield, M. Hilton, T. A. Marriage, G. E. Addison, Barrientos, and et al., The Atacama Cosmology Telescope: Sunyaev-Zel'dovich selected galaxy clusters at $148 \mathrm{GHz}$ from three seasons of data, Journal of Cosmology and Astroparticle Physics 7 8, [arXiv:1301.0816].

[32] D. S. Reed, R. E. Smith, D. Potter, A. Schneider, J. Stadel, and B. Moore, Towards an accurate mass function for precision cosmology, Montly Notices of the Royal Astronomical Society 431 1866-1882, [arXiv:1206.5302].

[33] M. Crocce, P. Fosalba, F. J. Castander, and E. Gaztañaga, Simulating the Universe with MICE: the abundance of massive clusters, Montly Notices of the Royal Astronomical Society 403 1353-1367, [arXiv:0907.0019].

[34] W. A. Watson, I. T. Iliev, A. D'Aloisio, A. Knebe, P. R. Shapiro, and G. Yepes, The halo mass function through the cosmic ages, Montly Notices of the Royal Astronomical Society 433 1230-1245, [arXiv: 1212.0095].

[35] G. Hinshaw, D. Larson, E. Komatsu, and et al., Nine-Year Wilkinson Microwave Anisotropy Probe (WMAP) Observations: Cosmological Parameter Results, Astrophysical Journal Supplement 208 19, [arXiv: 1212.5226].

[36] L. Anderson, É. Aubourg, S. Bailey, and et al., The clustering of galaxies in the SDSS-III Baryon Oscillation Spectroscopic Survey: baryon acoustic oscillations in the Data Releases 10 and 11 Galaxy samples, Montly Notices of the Royal Astronomical Society 441 24-62, [arXiv: 1312.4877].

[37] P. McDonald, U. Seljak, R. Cen, and et al., The Linear Theory Power Spectrum from the Lya Forest in the Sloan Digital Sky Survey, Astrophysical Journal 635 761-783, [astro-ph/0407377].

[38] A. Vikhlinin, R. A. Burenin, H. Ebeling, and et al., Chandra Cluster Cosmology Project. II. Samples and X-Ray Data Reduction, Astrophysical Journal 692 1033-1059, [arXiv: 0805.2207].

[39] K. Ichiki and M. Takada, Impact of massive neutrinos on the abundance of massive clusters, Physical Review D 85 063521, [arXiv:1108.4688].

[40] E. Castorina, E. Sefusatti, R. K. Sheth, F. Villaescusa-Navarro, and M. Viel, Cosmology with massive neutrinos II: on the universality of the halo mass function and bias, Journal of Cosmology and Astroparticle Physics 2 49, [arXiv:1311.1212].

[41] W. Cui, S. Borgani, and G. Murante, The effect of active galactic nuclei feedback on the halo mass function, Montly Notices of the Royal Astronomical Society 441 1769-1782, 
[arXiv: 1402.1493].

[42] R. Jimenez, T. Kitching, C. Peña-Garay, and L. Verde, Can we measure the neutrino mass hierarchy in the sky?, Journal of Cosmology and Astroparticle Physics 5 35, [arXiv: 1003.5918].

[43] G. Mention, M. Fechner, T. Lasserre, T. A. Mueller, D. Lhuillier, M. Cribier, and A. Letourneau, Reactor antineutrino anomaly, Physical Review D 83073006 , [arXiv:1101.2755].

[44] J. N. Abdurashitov, V. N. Gavrin, S. V. Girin, , and et al., Measurement of the response of a Ga solar neutrino experiment to neutrinos from a Ar37 source, Physical Review C $\mathbf{7 3} 045805$, [nucl-ex/0512041].

[45] C. Giunti, M. Laveder, Y. F. Li, Q. Y. Liu, and H. W. Long, Update of short-baseline electron neutrino and antineutrino disappearance, Physical Review D 86 113014, [arXiv:1210.5715].

[46] LSND Collaboration Collaboration, A. Aguilar-Arevalo and et al., Evidence for neutrino oscillations from the observation of anti-neutrino(electron) appearance in a anti-neutrino(muon) beam, Phys.Rev. D64 112007, [hep-ex/0104049].

[47] S. Hannestad, I. Tamborra, and T. Tram, Thermalisation of light sterile neutrinos in the early universe, Journal of Cosmology and Astroparticle Physics 7 25, [arXiv: 1204.5861].

[48] A. Melchiorri, O. Mena, S. Palomares-Ruiz, S. Pascoli, A. Slosar, and M. Sorel, Sterile neutrinos in light of recent cosmological and oscillation data: a multi-flavor scheme approach, Journal of Cosmology and Astroparticle Physics 1 36, [arXiv:0810.5133].

[49] S. Palomares-Ruiz, S. Pascoli, and T. Schwetz, Explaining LSND by a decaying sterile neutrino, Journal of High Energy Physics 9 48, [hep-ph/05].

[50] D. Hooper, F. S. Queiroz, and N. Y. Gnedin, Nonthermal dark matter mimicking an additional neutrino species in the early universe, Physical Review D 85 063513, [arXiv:1111.6599].

[51] C. Kelso, C. A. d. S. Pires, S. Profumo, F. S. Queiroz, and P. S. Rodrigues da Silva, A 331 WIMPy dark radiation model, European Physical Journal C $\mathbf{7 4}$ 2797, [arXiv:1308.6630].

[52] T. L. Smith, E. Pierpaoli, and M. Kamionkowski, New Cosmic Microwave Background Constraint to Primordial Gravitational Waves, Physical Review Letters 97 021301, [astro-ph/].

[53] E. Calabrese, D. Huterer, E. V. Linder, A. Melchiorri, and L. Pagano, Limits on dark radiation, early dark energy, and relativistic degrees of freedom, Physical Review D $\mathbf{8 3}$ 123504, [arXiv:1103.4132].

[54] P. Langacker, ON THE COSMOLOGICAL PRODUCTION OF LIGHT STERILE-NEUTRINOS, (University of Pennsylvania, 1989).

[55] S. Dodelson and L. M. Widrow, Sterile neutrinos as dark matter, Physical Review Letters 72 17-20, [hep-ph/9303287].

[56] A. Lewis and S. Bridle, Cosmological parameters from CMB and other data: A Monte Carlo approach, Physical Review D 66 103511, [astro-ph/].

[57] Planck collaboration, Planck 2013 results. XV. CMB power spectra and likelihood, ArXiv e-prints [arXiv: 1303.5075].

[58] J. Lesgourgues, M. Viel, M. G. Haehnelt, and R. Massey, A combined analysis of 3D weak lensing, Lyman- $\alpha$ forest and WMAP year three data, Journal of Cosmology and Astroparticle Physics 11 8, [arXiv:0705.0533].

[59] S. Bridle and L. King, Dark energy constraints from cosmic shear power spectra: impact of intrinsic alignments on photometric redshift requirements, New Journal of Physics $\mathbf{9} 444$, [arXiv:0705.0166]. 
[60] N. Palanque-Delabrouille, C. Yèche, A. Borde, and et al., The one-dimensional Lya forest power spectrum from BOSS, Astronomy and Astrophysics 559 A85, [arXiv:1306.5896].

[61] U. Seljak, A. Slosar, and P. McDonald, Cosmological parameters from combining the Lyman- $\alpha$ forest with CMB, galaxy clustering and SN constraints, Journal of Cosmology and Astroparticle Physics 10 14, [astro-ph/].

[62] A. Borde, N. Palanque-Delabrouille, G. Rossi, M. Viel, J. S. Bolton, C. Yèche, J.-M. LeGoff, and J. Rich, New approach for precise computation of Lyman- $\alpha$ forest power spectrum with hydrodynamical simulations, Journal of Cosmology and Astroparticle Physics $\mathbf{7} 5$, [arXiv: 1401.6472].

[63] G. Rossi, N. Palanque-Delabrouille, A. Borde, M. Viel, C. Yèche, J. S. Bolton, J. Rich, and

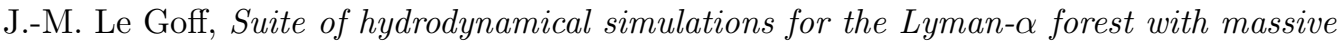
neutrinos, Astronomy and Astrophysics 567 A79, [arXiv:1401.6464].

[64] A. V. Kravtsov, A. Vikhlinin, and D. Nagai, A New Robust Low-Scatter X-Ray Mass Indicator for Clusters of Galaxies, Astrophysical Journal 650 128-136, [astro-ph/0603205].

[65] J. Tinker, A. V. Kravtsov, A. Klypin, K. Abazajian, M. Warren, and et al., Toward a Halo Mass Function for Precision Cosmology: The Limits of Universality, Astrophysical Journal 688 709-728, [arXiv:0803.2706].

[66] R. A. Burenin and A. A. Vikhlinin, Cosmological parameters constraints from galaxy cluster mass function measurements in combination with other cosmological data, Astronomy Letters 38 347-363, [arXiv:1202.2889].

[67] J. Brandbyge, S. Hannestad, T. Haugbølle, and Y. Y. Y. Wong, Neutrinos in non-linear structure formation - the effect on halo properties, Journal of Cosmology and Astroparticle Physics 9 14, [arXiv: 1004.4105].

[68] F. Marulli, C. Carbone, M. Viel, L. Moscardini, and A. Cimatti, Effects of massive neutrinos on the large-scale structure of the Universe, Montly Notices of the Royal Astronomical Society 418 346-356, [arXiv:1103.0278].

[69] F. Villaescusa-Navarro, S. Bird, C. Peña-Garay, and M. Viel, Non-linear evolution of the cosmic neutrino background, Journal of Cosmology and Astroparticle Physics 3 19, [arXiv: 1212.4855].

[70] M. Costanzi, F. Villaescusa-Navarro, M. Viel, J.-Q. Xia, S. Borgani, E. Castorina, and E. Sefusatti, Cosmology with massive neutrinos III: the halo mass function and an application to galaxy clusters, Journal of Cosmology and Astroparticle Physics 12 12, [arXiv:1311.1514].

[71] A. Jenkins, C. S. Frenk, S. D. M. White, J. M. Colberg, S. Cole, A. E. Evrard, H. M. P. Couchman, and N. Yoshida, The mass function of dark matter haloes, Montly Notices of the Royal Astronomical Society 321 372-384, [astro-ph/].

[72] D. S. Reed, R. Bower, C. S. Frenk, A. Jenkins, and T. Theuns, The halo mass function from the dark ages through the present day, Montly Notices of the Royal Astronomical Society $\mathbf{3 7 4}$ $2-15$, [astro-ph/].

[73] M. S. Warren, K. Abazajian, D. E. Holz, and L. Teodoro, Precision Determination of the Mass Function of Dark Matter Halos, Astrophysical Journal 646 881-885, [astro-ph/].

[74] S. J. Cusworth, S. T. Kay, R. A. Battye, and P. A. Thomas, Impact of baryons on the cluster mass function and cosmological parameter determination, Montly Notices of the Royal Astronomical Society 439 2485-2493, [arXiv: 1309.4094].

[75] M. Velliscig, M. P. van Daalen, J. Schaye, I. G. McCarthy, M. Cacciato, A. M. C. Le Brun, and C. D. Vecchia, The impact of galaxy formation on the total mass, mass profile and abundance of haloes, Montly Notices of the Royal Astronomical Society 442 2641-2658, [arXiv:1402.4461]. 
[76] M. Vogelsberger, S. Genel, V. Springel, P. Torrey, D. Sijacki, D. Xu, G. F. Snyder, D. Nelson, and L. Hernquist, Introducing the Illustris Project: Simulating the coevolution of dark and visible matter in the Universe, ArXiv e-prints [arXiv:1405.2921].

[77] D. Martizzi, I. Mohammed, R. Teyssier, and B. Moore, The biasing of baryons on the cluster mass function and cosmological parameter estimation, Montly Notices of the Royal Astronomical Society 440 2290-2299, [arXiv: 1307.6002].

[78] A. Mahdavi, H. Hoekstra, A. Babul, and J. P. Henry, Evidence for non-hydrostatic gas from the cluster X-ray to lensing mass ratio, Montly Notices of the Royal Astronomical Society $\mathbf{3 8 4}$ 1567-1574, [arXiv:0710.4132].

[79] E. Rasia, M. Meneghetti, R. Martino, S. Borgani, A. Bonafede, K. Dolag, S. Ettori, D. Fabjan, C. Giocoli, P. Mazzotta, J. Merten, M. Radovich, and L. Tornatore, Lensing and x-ray mass estimates of clusters (simulations), New Journal of Physics 14 055018, [arXiv:1201.1569].

[80] T. F. Laganá, R. S. de Souza, and G. R. Keller, On the influence of non-thermal pressure on the mass determination of galaxy clusters, Astronomy and Astrophysics $\mathbf{5 1 0}$ A76, [arXiv:0911.0647].

[81] X. Shi and E. Komatsu, Analytical model for non-thermal pressure in galaxy clusters, ArXiv e-prints [arXiv:1401.7657].

[82] H. Israel, T. H. Reiprich, T. Erben, R. J. Massey, C. L. Sarazin, P. Schneider, and A. Vikhlinin, The 400d Galaxy Cluster Survey weak lensing programme. III. Evidence for consistent WL and $X$-ray masses at $z \approx 0.5$, Astronomy and Astrophysics 564 A129, [arXiv:1402.3267].

[83] M. Donahue, G. M. Voit, A. Mahdavi, and et al., CLASH-X: A Comparison of Lensing and X-ray Techniques for Measuring the Mass Profiles of Galaxy Clusters, ArXiv e-prints [arXiv: 1405.7876].

[84] A. von der Linden, A. Mantz, S. W. Allen, and et al., Robust Weak-lensing Mass Calibration of Planck Galaxy Clusters, ArXiv e-prints [arXiv:1402.2670].

[85] J. Nevalainen, L. David, and M. Guainazzi, Cross-calibrating X-ray detectors with clusters of galaxies: an IACHEC study, Astronomy and Astrophysics 523 A22, [arXiv: 1008.2102].

[86] G. Schellenberger, T. H. Reiprich, L. Lovisari, J. Nevalainen, and L. David, XMM-Newton and Chandra Cross Calibration Using HIFLUGCS Galaxy Clusters, ArXiv e-prints [arXiv: 1404.7130].

[87] A. Gelman and D. B. Rubin, Inference from iterative simulation using multiple sequences, Statistical Science 7 457-472.

[88] D. Spergel, R. Flauger, and R. Hlozek, Planck Data Reconsidered, ArXiv e-prints [arXiv: 1312.3313].

[89] A. B. Mantz, A. von der Linden, S. W. Allen, and et al., Weighing the Giants IV: Cosmology and Neutrino Mass, ArXiv e-prints [arXiv:1407.4516]. 